\title{
Somatic HCN channels augment and speed up GABAergic basket cell input-output function in human neocortex
}

Viktor Szegedi ${ }^{1}$, Emőke Bakos ${ }^{1}$, Szabina Furdan ${ }^{1}$, Pal Barzo $^{2}$, Gabor Tamas $^{3}$ and Karri Lamsa ${ }^{1 *}$

${ }^{1}$ MTA-NAP Research Group for Inhibitory Interneurons and Plasticity, Department of Physiology, Anatomy and Neuroscience, University of Szeged, Közép fasor 52. Szeged, 6726 Hungary

${ }^{2}$ Department of Neurosurgery, University of Szeged, Semmelweis u. 6.6725 Szeged, Hungary ${ }^{3}$ MTA-SZTE Research Group for Cortical Microcircuits, Department of Physiology, Anatomy and Neuroscience, University of Szeged, Közép fasor 52. Szeged, 6726 Hungary

*Correspondence to: Karri Lamsa, email: klamsa@bio.u-szeged.hu

Running title: Somatic boost in human inhibitory basket cells

\section{ABSTRACT}

Neurons in the mammalian brain exhibit evolution-driven species-specific differences in their functional properties. Therefore, understanding the human brain requires unraveling the human neuron "uniqueness" and how it contributes to the operation of specific neuronal circuits. We show here that a highly abundant type of inhibitory neurons in the neocortex, GABAergic parvalbumin-expressing basket cell (pv+BC), exhibits in the human brain a specific somatic leak current mechanism, which is absent in their rodent neuronal counterparts. Human pv+BC soma shows electric leak conductance mediated by hyperpolarization-activated cyclic nucleotide-gated channels. This leak conductance has depolarizing effects on the resting membrane potential and it accelerates the rise of synaptic potentials in the cell soma. The leak facilitates the human pv+BC input-to-output fidelity and shortens the action potential generation to excitatory inputs. This mechanism constitutes an adaptation that enhances signal transmission fidelity and speed in the common inhibitory circuit in the human but not in the rodent neocortex. 


\section{INTRODUCTION}

Fast-spiking GABAergic interneurons of the mammalian cerebral cortex play central role in cognitive and sensory processes by supplying accurately timed inhibition of neuronal networks ${ }^{1,2}$. Precise and faithful operation of these parvalbumin-expressing $(p v+)$ neurons relies on "fast in-fast out" function, by which they transform excitatory synaptic inputs to inhibitory outputs with a high fidelity, short latency and sub-millisecond temporal precision (see for instance ${ }^{3}$ ). Time-lag and jitter in their operation mostly arise from the transformation of excitatory postsynaptic potentials (EPSPS) into a spiking output ${ }^{4,5}$. This EPSP-spike transformation takes place in (or very close to) cell soma and the process is welldocumented in rodent cortical neurons including the pv+ GABAergic cell population ${ }^{1,6,7}$. Yet very few studies have investigated the EPSP-spike transformation or the input-to-output function in human neurons $\mathrm{s}^{8,9}, 10,11,12,13$ and particularly the GABAergic human neuronal types have received little attention. Understanding the functioning of human neurons is highly relevant, because findings in experimental animals do not always translate to human. In fact, analogous neuronal types between the human and rodent cortex exhibit various between-species differences in their physiological functional parameters 9 , 13, 14, 15, 16, 17, 18, 19, ${ }^{20,21}$. Species-specific behaviors may hence arise from even small differences in neuronal types and neuronal circuits ${ }^{22,23}$, making it critical to uncover the "uniqueness" of the human neurons and to investigate its outcome on the operation of anatomically identified neuronal circuits.

Although single-cell RNA sequencing studies denote relatively conserved gene expression patterns among GABAergic inhibitory neurons of the mammalian neocortex ${ }^{24,25}$, analogous 
neuronal types such as pv+ GABAergic interneurons show inter-species differences in terms of neurite arborization ${ }^{26}$, ion channel expression ${ }^{27}$, as well as temporal dimensions of basal synaptic transmission ${ }^{14,28}$. In general, pv+ cortical interneurons are characterized by rapid action potentials and a high-frequency firing capacity across various experimental species ${ }^{10,}$ $29,30,31,32$, but action potential firing and intrinsic electrical properties of the cells differ quantitatively between species ${ }^{28,31,33}$. However, it remains unknown how these affect EPSP-spike transformation and input-to-output function of the most numerous neocortical inhibitory interneuronal type, the pv+ basket cell $(B C)^{34,35}$ in human.

Excitability in cell soma in many neurons is enhanced by hyperpolarization-activated cyclic nucleotide-gated ( $\mathrm{HCN}$ ) channels and these provide a well-documented mechanism allowing to facilitate EPSP kinetics and the EPSP-spike coupling $33,36,37,38,39$. HCN channels are voltage-gated cation channels opening at potentials negative to $-50 \mathrm{mV}$ that regulate resting membrane potential and intrinsic excitability by their permeability to $\mathrm{K}^{+}$and $\mathrm{Na}^{+}$ ions, with a reversal potential of approximately $-30 \mathrm{mV}{ }^{40,41}$. RNA sequencing studies and protein analyses indicate high levels of $\mathrm{HCN}$-channel expression in the human and rodent neocortex ${ }^{18,42}$. In neocortical pv+ GABAergic neurons, which contain primarily the HCNchannel type 1 (HCN1) subunit $40,43,44,45$, rodent experiments showed their absence in the soma. Although pv+ neurons in some other areas of brain exhibit somatic HCN-channel activity ${ }^{40,46,47}$, in the neocortex the HCN channels are localized in their axon ${ }^{42,48,49}$. This is obvious in many studies demonstrating minuscule hyperpolarization-evoked voltage sag potential, which is a hallmark of somatic $\mathrm{HCN}$ activity, in neocortical pv+ cells. Furthermore, $\mathrm{HCN}$-channel blockers barely have any effect on the rodent neocortical pv+ cell resting 
membrane potential or somatic excitability $33,36,37,38,39,43,50$. Nevertheless, HCN-channel activity and its localization in human pv+ neurons have remained elusive.

We investigated somatic $\mathrm{HCN}$-channel activity and its role in the transformation of excitatory inputs into a spike in human pv+BCs among neocortical layers $2 / 3$ (L2/3) where neurons make major contributions to corticocortical connections and function to integrate information among cortical areas (for instance see ${ }^{51}$ ). The study was performed using acute human brain slices from non-pathological neocortical tissue resected in deep area brain surgery. We report robust $\mathrm{HCN}$-channel activity in the somatic compartment of human, but not mouse, anatomically-identified parvalbumin-immunopositive BCs. Somatic HCN conductance depolarizes the resting potential, reduces excitatory synaptic potential (EPSP) onset-to-peak time, and shortens the delay from excitatory somatic currents to spike in human interneurons. These findings indicate that somatic $\mathrm{HCN}$-channel activity promotes signal transmission through this anatomically-identified inhibitory neuronal circuit in the human neocortex. We suggest that this mechanism represents an evolutionary adaptation to increase fidelity, speed, and precision of signal transmission through this common inhibitory circuit in the human neocortex.

\section{RESULTS}

We studied 45 pv+BCs in the human neocortex $L 2 / 3$ to assess $\mathrm{HCN}$-channel mediated activity in their soma. Cells were recorded in whole-cell clamp within acute neocortical slices prepared from tissue material that had to be removed in surgery to gain access to deeperbrain targets. Cells were visualized and identified by their axon forming boutons around 
unlabeled $L 2 / 3$ neurons, and by positive immunoreaction for $p v(p v+; n=45)$. For comparison, we studied 38 visualized fast-spiking BCs in the mouse somatosensory cortex (Supplementary Table 1).

pv+BCs show large HCN-channel-mediated somatic sag potential in human but not in mouse

We found that $\mathrm{pv}+\mathrm{BCs}$ in the human neocortex show robust hyperpolarization-activated sag potential. First, we recorded cells in current clamp by applying square-pulse current steps (250 ms) to hyperpolarize or depolarize the membrane potential (range from $-95 \mathrm{mV}$ to +40 $\mathrm{mV}$ ) at a holding potential of $-70 \mathrm{mV}$ (Fig. 1A1-2). The hyperpolarizing square-pulse steps evoked voltage sag during the step, and a rebound voltage sag of similar amplitude but opposite polarity was measured following the membrane potential step ${ }^{18}(P=0.516$, Mann-Whitney U-test) (Supplementary Table 2). This property is characteristic of HCNchannel activation during hyperpolarization and of $\mathrm{HCN}$-channel inactivation by repolarization, respectively ${ }^{18,40}$ (see Fig. 1A1-2). In addition, the HCN-channel antagonist ZD7288 (10 $\mu \mathrm{M})$ similarly blocked the voltage sag and the rebound sag (Fig. 1A3).

We next compared somatic sag robustness between human and mouse pv+BCs by using hyperpolarizing voltage step at $-70 \mathrm{mV}$. In human ( $\mathrm{n}=28$ cells), hyperpolarizing step to -90.6 $\mathrm{mV}$ (average) showed a $2.91 \mathrm{mV}$ average sag potential amplitude, with a large range observed between cells from 0 to $15 \mathrm{mV}$. In mouse ( $\mathrm{n}=32$ cells), a similar hyperpolarizing step (to $-86.4 \mathrm{mV}$ average) showed $0.53 \mathrm{mV}$ sag, with values ranging from 0 to $3.0 \mathrm{mV}$ (Fig. 1B1). A difference in sag amplitude between human and mouse cells was also seen when a hyperpolarizing membrane potential step was applied to $-70 \mathrm{mV}$ at $-50 \mathrm{mV}$ (Fig. 1B2). 
Hyperpolarization of human $\mathrm{pv}+\mathrm{BCs}$ from $-49.3 \mathrm{mV}$ (average) to $-70.5 \mathrm{mV}$ revealed a 1.9 $\mathrm{mV}$ average sag with a range from 0 to $9.5 \mathrm{mV}$. Correspondingly, in mouse pv+BCs, a step from $-46.1 \mathrm{mV}$ to $-71.0 \mathrm{mV}$ on average showed a barely detectable sag of just $0.2 \mathrm{mV}$ on average with a range from 0 to $2 \mathrm{mV}$. Comparing between human and mouse pv+BCs further showed a statistically significant difference in somatic voltage sag amplitude at both step protocols ( $P<0.001$, Mann-Whitney U-test) (Fig. 1B1-2).

The pv+BCs were systematically filled with biocytin during recording and their anatomical location was determined post hoc (Fig. 1C1). We therefore examined the vertical position of pv+BCs soma in $\mathrm{L} 2 / 3$ to assess whether the voltage sag strength differed between cells localized in the superficial or deep L2/3 (Fig. 1C2). Yet we failed to find correlation between sag amplitude and the depth of cell soma in $L 2 / 3(P=0.298$, correlation coefficient $=0.207$, Spearman correlation). The soma location for each cell was measured as their relative positioning from the L1 border to the L4 border (see Fig. 1C2) (see Methods).

\section{$\mathrm{HCN}$ channels depolarize somatic resting membrane potential in human pv+BCs}

We next studied somatic HCN-channel activity in 18 randomly selected human and in 18 randomly selected mouse pv+BCs that were kept at resting membrane potential (Em). By applying a robust depolarizing subthreshold step to $-50 \mathrm{mV}(250 \mathrm{~ms}$, average in human = $-48.1 \mathrm{mV}$, mouse $=-46.0 \mathrm{mV}, \mathrm{n}=18$ and 18$)$, we aimed to inactivate $\mathrm{HCN}$ channels, which are known to reactivate by repolarization to $\mathrm{Em}$ and to generate a voltage sag ${ }^{40,42}$. Figure $2 \mathrm{~A}$ summarizes the results showing the Em of human and mouse pv+BCs (Fig. 2A1) and the voltage sag amplitude that was measured at Em in these cells following the depolarizing step (Fig. 2A2). 
Altogether, human cells showed a $2.20 \mathrm{mV}$ average sag potential amplitude with a range from 1.0 to $5.1 \mathrm{mV}$ at their Em (average $-66.3 \mathrm{mV}, \mathrm{n}=18$ ). For comparison, the identified pv+BCs in mouse showed a $0.66 \mathrm{mV}$ average sag amplitude with a range from 0 to $1.7 \mathrm{mV}$ at Em (average $-61.2 \mathrm{mV}, \mathrm{n}=18$ ) (Fig. 1B1). Subtracting the voltage sag amplitude from the Em value measured at steady-state (see Fig. $2 \mathrm{~A} 2$ inset) revealed the depolarizing effect of $\mathrm{HCN}$-channel activity on their somatic membrane potential under resting conditions. Figure 2B2 sums up this depolarizing effect on Em in both human and mouse pv+BCs. However, somatic HCN activity fails to explain the diversity of Em values observed between individual pv+BCs (Supplementary Fig. S1).

In addition, the depolarizing effect of $\mathrm{HCN}$-channel activity on Em was measured in other set of experiments, in which ZD7288 (ZD; $10 \mu \mathrm{M})$ was washed-in while monitoring Em in pv+BCs. In 11 human and 7 mouse pv+BCs, we measured Em just before ZD application and after 3-5 min in presence of the drug. We found a $-5.65 \mathrm{mV}$ average shift of Em (in control $-61.9 \pm 6.9 \mathrm{mV})$ in human $\mathrm{pv}+\mathrm{BCs}(\mathrm{P}=0.006)$ compared to a $-0.69 \mathrm{mV}$ average change in mouse pv+BCs $(n=7$, in control $-69.0 \pm 12.9 \mathrm{mV})(P=0.261)$ (paired Student's t-test). The Em data in control conditions and in the presence $\mathrm{ZD}$ are summarized in Figure $2 \mathrm{~B} 3$. The relatively small sample size in these experiments is explained by fact that in most recordings with ZD, Em was not monitored during ZD application.

\section{HCN channels maintain somatic leak conductance in human pv+BCs at resting potential}

In human pv+BCs, we obtained robust immunohistochemical staining for HCN1, the common channel isoform and the major HCN-channel protein in cortical neurons $40,43,45$. 
We performed double immunohistochemical labeling for pv and HCN1 in three human tissue samples (Fig. 3A). The immunohistochemical results show HCN1 in human pv+BCs. The results support our electrophysiological findings described above in current clamp showing HCN-channel activity in the somatic compartment in human pv+BCs. Indeed, we verified $\mathrm{HCN}$ channel-mediated electric conductance in human pv+BC soma. We performed voltage-clamp recording in five human and five mouse pv+BCs that were selected randomly for the experiments. We voltage-clamped the cells at their Em (average in human of -59.0 $\mathrm{mV}$, mouse of $-70.6 \mathrm{mV}$ ), and measured somatic leaking conductance (Gleak) by using brief voltage-clamp steps (-10 or $10 \mathrm{mV}, 10 \mathrm{~ms}$ applied at $0.1 \mathrm{~Hz}$ ) while washing-in the HCNblocker ZD (10 $\mu \mathrm{M})$. In human pv+BCs, Gleak was 3.78 nS, 2.97-4.46 nS (median, quartiles) in baseline control conditions and $3.32 \mathrm{nS}, 2.31-3.88 \mathrm{nS}$ following application of ZD (3-5 min). Thus, ZD reduced Gleak to 0.84 (average, range 0.57 to 1.04 ) from baseline control. In mouse pv+BCs, Gleak was $4.87 \mathrm{nS}, 4.75-6.17 \mathrm{nS}$ in baseline control conditions. In the presence of ZD, baseline-normalized Gleak was 0.96 (average, range 0.94 to 0.98 ). The ZDsensitive conductance generated through $\mathrm{HCN}$ channels $\left(\mathrm{G}_{\mathrm{HCN}}\right)$ was of $0.58 \mathrm{nS}$ (average range of $0-1.42 \mathrm{nS}$ ) in the human pv+BCs, and of $0.21 \mathrm{nS}$ (average range of $0.12-0.28 \mathrm{nS}$ ) in the mouse $\mathrm{pv}+\mathrm{BCs}$. Figure $3 \mathrm{~B}$ and $\mathrm{C}$ illustrate the conductance measurement made in these experiments. Figure 3D summarizes the measured leaking conductance in all pv+BCs in control conditions and in the presence of ZD. Leaking conductance was measured from the voltage step current at the end of step when it approached a steady-state level, while the amplitude is in a linear relation to the soma leaking conductance.

HCN-channel leak conductance shortens the membrane time-constant, the EPSP time-topeak and the EPSC-to-spike transformation in human pv+BCs 
Considering the evidence presented above of $\mathrm{HCN}$-channel activity in human pv+BC soma, we studied the effect of a HCN-channel blocker on somatic membrane time constant in human and mouse pv+BCs. To measure the effect of ZD $(10 \mu \mathrm{M})$ on passive membrane time constant (membrane tau) in pv+BCs, we applied hyperpolarizing voltage steps at $-70 \mathrm{mV}$ (Fig. 4A1), and found that the drug wash-in was associated with a 1.33-time (average range of 1.08-1.63 in ZD compared to baseline control) prolongation of the membrane tau in human $\mathrm{pv}+\mathrm{BCs}$, and a 1.14-time (average range of $0.98-1.28$ ) prolongation in mouse pv+BCs (Fig. 4A2) ( $P=0.059$, Student's t-test). Membrane tau for human cells was $6.76 \mathrm{~ms}, 6.55-$ $8.22 \mathrm{~ms}$ in control conditions (median and quartiles, $\mathrm{n}=8$ ), and $9.72 \mathrm{~ms}, 8.15-10.53 \mathrm{~ms}$ in ZD conditions ( $P=0.008$, Wilcoxon Signed-Rank Test), when measured in standard $20 \mathrm{mV}$ hyperpolarizing steps at $-70 \mathrm{mV}$ as illustrated in Figure $4 \mathrm{~A} 1$ inset. Correspondingly, tau values in mouse pv+BCs were $5.56 \mathrm{~ms}, 5.33-6.16 \mathrm{~ms}$ in control and $6.95 \mathrm{~ms}, 5.40-7.42 \mathrm{in} \mathrm{ZD}$ ( $n=7)(P=0.031$, Wilcoxon Signed-Rank Test). Importantly, the effect of ZD on membrane tau was most pronounced in pv+BCs showing the largest $\mathrm{HCN}$ voltage sag potentials in control conditions before the ZD wash-in ( $P>0.001, n=15$ cells, Pearson's correlation). Cross-correlation of the two ZD-sensitive variables is illustrated in Figure 4A3. Together, these results indicate that $\mathrm{HCN}$-channel blocker affects somatic passive membrane time constant more pronouncedly in human than in mouse pv+BCs.

By using dynamic-clamp, a computer-driven current injection system mimicking synaptic currents, we next applied somatic excitatory postsynaptic currents (EPSCS) similar to those occurring in human during pyramidal cell-to-pv+BC communication ${ }^{14,}{ }^{15}$, in randomly selected separate sets of $\mathrm{pv}+\mathrm{BC}$ in human and mouse. In cells recorded at $-70 \mathrm{mV}$, we evoked large subthreshold EPSPS nearly reaching the action potential firing threshold. The 
results show that HCN-channel blocker ZD prolongs the time-to-peak of the EPSPS generated by the EPSCS (Fig. 4B1). By comparing EPSPS of similar peak amplitude in the control condition and in the presence of ZD, the EPSP time-to-peak was prolonged with the drug in human $(P=0.004, n=9)$ and also in mouse $(P=0.029, n=8)$ cells (paired Student's t-test) (Fig. 4B2). However, the effect of ZD on the EPSP time-to-peak was found to be larger in human compared to mouse pv+BCs $(P=0.049$, Student's t-test; Shapiro-Wilk normality $P$ $=0.34$ ), increasing the baseline-normalized value to $1.18 \pm 0.14$ in human and to $1.07 \pm 0.06$ in mouse pv+BCs.

Given that ZD prolongs the membrane tau and the EPSP time-to-peak in human pv+BC soma, we continued our investigation using dynamic-clamp to study the effect of HCNchannel blockade on EPSC transformation to a spike in pv+BCs. By applying incrementally increasing EPSC amplitudes (testing 4-5 different EPSC strengths) to elicit EPSPS with the peak amplitude reaching the firing threshold, we assessed the time lag for the EPSC transformation to an action potential in control conditions and after a brief wash-in ( $5 \mathrm{~min}$ ) with ZD. Figure 5A illustrates the sample dynamic-clamp EPSC, the EPSP generated and the plots data on the EPSC-to-spike delay relationship in one pv+BC.

Because ZD increases EPSP amplitude by EPSCS, but not the relation of EPSP amplitude and spiking probability (Supplementary Fig. S2-S3), we compared the spike delay between similar amplitude EPSPs in the control and ZD conditions. Figure 5B1 illustrates one experiment in human pv+BCs with EPSP-spike probability close to 0.5. In the presence of ZD, we set EPSC strength to evoke EPSP with spiking at a similar probability as in the control condition. The probability was set to $0.5<1$. To eliminate Em shift caused by ZD, and thus, 
to focus on the HCN-channel effect on the somatic membrane time constant and lag in the spike generation, the membrane potential was held at $-70 \mathrm{mV}$ throughout the experiments. We found that the EPSC-to-spike delay increased in five out of six human pv+BCs but only in one out of five mouse pv+BC. The results are illustrated in Figures 5B2-C. Importantly, we compared the spike-delay in ZD (normalized by spike delay in control) with the cell's passive membrane time constant in ZD (normalized by the value in control conditions), revealing a strong correlation between them ( $P=0.013$, Pearson's test) (Fig. 5C).

\section{HCN-channel conductance in soma facilitates pv+BC input-to-output function}

Finally, we investigated the effect of somatic $\mathrm{HCN}$ conductance on $p v+B C s$ in a computational model. We used a single-cell soma model (NEURON-7) with passive membrane properties to simulate the effect of $\mathrm{G}_{\mathrm{HCN}}$ on the cell Em, the EPSP amplitude and the EPSP time-to-peak at the same time, since these features were studied separately in the experiments above. We simulated the five human and five mouse pv+BCs with cell capacitance $(\mathrm{Cm})$, Em measured in control conditions, total leaking conductance measured in control conditions, and the ZD-sensitive leaking conductance (measured in the cells presented in Fig. 3 and summarized in Supplementary Table 3). To elicit the EPSPS, we used same EPSC parameters as in the dynamic-clamp experiments above (see Figs. 4-5). The simulation demonstrated a strong faciliatory effect of the $G_{H C N}$ in human pv+BCs, with the conductance values occurring naturally in these cells (Fig. 6A). Importantly, the simulations demonstrate that although the somatic HCN conductance leads to an attenuated EPSP amplitude in $\mathrm{pv}+\mathrm{BCs}$, the simultaneous depolarization of $\mathrm{Em}$ by somatic $\mathrm{G}_{\mathrm{HCN}}$-mediated depolarizing leaking current (with a reversal potential at $-30 \mathrm{mV}$ ) ${ }^{40,42}$ overwhelms the EPSP amplitude attenuation. Thus, in all cells with natural $G_{H C N}$ measured in their soma, this 
conductance promotes EPSC-to-spike transformation; it allows for a somatic excitatory current to reach higher depolarization level from $\mathrm{Em}$, and in addition it facilitates the EPSP time-to-peak to reach any depolarized threshold level with a shorter delay. Figure 6A1-3 illustrates the effects of $G_{H C N}$ in three human pv+BCs. Correspondingly, ZD-sensitive conductance measured in mouse pv+BC soma had no effect or only a small effect on cell Em or EPSP. Figure 6B1-3 shows the simulated EPSPS at Em in three mouse pv+BCs. Thus, the simulations demonstrate that the net effect of somatic HCN conductance is a facilitation of the excitatory input-to-output function (Fig. 6C1-3), which speeds up the EPSC-EPSP transformation to reach firing threshold in these cells (Fig. 6D).

\section{DISCUSSION}

Our results reveal robust somatic $\mathrm{HCN}$-channel activity in pv+BCs of the human neocortex. The somatic $\mathrm{HCN}$-channel responses are absent in pv+BCs of the mouse neocortex, as was reported in various earlier studies $33,36,37,38,39,43,50$. These findings demonstrate, together with the computational modeling, how "a human-specific" physiological feature augments and speeds up signal transfer in the highly abundant inhibitory circuit in neocortex. This emphasizes the importance of conducting physiological studies in human identified cell types in order to uncover their "uniqueness."

\section{Between-species difference in somatic HCN activity alters basket cell physiological} function

$\mathrm{HCN}$ channels in the somatic compartment modulate the entire human pv+BC function through the leaking conductance at potentials more negative than $-50 \mathrm{mV}$. The $\mathrm{HCN}-$ 
mediated somatic conductance facilitates their EPSP rise, and although it reduces the EPSP peak amplitude, it depolarizes the resting potential more than it reduces the EPSP amplitude. A similar net effect of $\mathrm{HCN}$ leak conductance was reported in glutamatergic neurons in rodent and human ${ }^{18,52,53}$. We show here that the somatic HCN facilitates inputto-output function in the most abundant neocortical interneuron type in human but not mouse.

The somatic membrane time-constant is a key determinant of the input-output relation of neurons and it determines how rapidly a neuron responds to an excitatory stimulus ${ }^{52}$. In the human neocortex, unitary pyramidal cells form powerful excitatory connections on local pv+BCs, in order to generate suprathreshold EPSPs at the interneuron resting potential by unitary spike ${ }^{10,14,15,21}$. In this input-to-output signaling, the pv+BC's firing delay to the large EPSC is couple of milliseconds ${ }^{10,12,2154}$. In the rapid EPSP-spike transformation, the somatic $\mathrm{G}_{\mathrm{HCN}}$ at $\mathrm{Em}$ is highly relevant, considering that $\mathrm{HCN}$-channel conductance remains practically unchanged during fast Vm shifts such as EPSP rise and the EPSP-spike coupling. Although $\mathrm{G}_{\mathrm{HCN}}$ depends on the membrane potential, it changes relatively slowly by membrane potential shifts with a time constant of $20-50 \mathrm{~ms}^{40,55}$.

Together, our results demonstrate that with the contribution of somatic HCN, human pv+BCs keep their time-constant and EPSP time-to-peak kinetics to a similar level as in rodent cells. Without the somatic $\mathrm{G}_{\mathrm{HCN}}$, human cells would be substantially slower than their rodent counterparts. Thus, $\mathrm{G}_{\mathrm{HCN}}$ activity appears critical for the rapid membrane potential dynamics in human pv+BCs. 


\section{Rationale of basket cell specialization is efficiency}

Although benefits of somatic $\mathrm{G}_{\mathrm{HCN}}$ in neuronal signaling are obvious, a question remains: why has this mechanism evolved in human (and presumable in other primate) neocortical pv+BCs but not in the same cell type in rodent neocortex?

Firstly, we speculate that in general there is evolutionary pressure toward faster and higher fidelity signaling in neuronal pathways ${ }^{22}$. Secondly, many human neurons including the pv+BCs exhibit highly resistive cell membrane and a relatively large cell capacitance, which together result in slow passive membrane time constant and EPSP-to-spike transformation in cell soma ${ }^{19,28,31}$. This was observed here in the experiments blocking HCN channels. Thus, the active leak conductance from somatic $\mathrm{G}_{\mathrm{HCN}}$ channels appears critical in human cells to speed up their membrane potential dynamics. For some unknown reason, the phylogenetic development of $B C$ intrinsic properties has followed a different path in the rodent neocortex, since these cells lack or show very small somatic $\mathrm{G}_{\mathrm{HCN}}$ although they have prominent somatic leaking conductance ${ }^{33,36,43}$. Apparently, rodent pv+BCs maintain their somatic leakiness (and reduce their membrane time constant) through $\mathrm{K}^{+}$channels and voltage-gated $\mathrm{Cl}^{-}$channel conductance instead ${ }^{4,56}$. Interestingly, like human pv+BCs, fastspiking neocortical basket cells of macaque monkeys exhibit high input resistance, a relatively long passive membrane time constant, and a large HCN channel-mediated voltage sag, suggesting robust somatic HCN-channel activity ${ }^{33,37,50}$. Thus, somatic HCN-channel leak conductance in neocortical pv+BCs may be a general feature encountered across primates ${ }^{57}$ which separated from rodents 70-90 million years ago ${ }^{58}$. 
Overall, these results support the need for more functional studies with quantitative analysis of the physiological parameters of identified human neuron types. This knowledge is required to uncover the human "uniqueness" across various neuroanatomical circuits. By employing computational models, the generated data will help us to understand why species-specific neuronal features are important and relevant to cortical processes. Some of these features may eventually turn out to be key targets for neurological and neuropsychiatric disorders as well as neurodegenerative diseases ${ }^{16,59,60,61,62}$.

FIGURE LEGENDS

Figure 1. Human compared to mouse pv+BCs show a robust somatic HCN-channelmediated sag potential

A1. Human $\mathrm{pv}+\mathrm{BC}$ somatic membrane potential responses to depolarizing or hyperpolarizing square-pulse current steps delivered at $-70 \mathrm{mV}$. Traces in control (blue) show characteristic hyperpolarization-activated sag potential and its blockade by ZD7288 (ZD; $10 \mu \mathrm{M}$ ) (black traces). One trace in control conditions is shown in red to illustrate the ZD-sensitive sag potential amplitude during a hyperpolarizing step, and rebound sag at resting membrane potential (Em) following the step (scale $10 \mathrm{mV}, 100 \mathrm{~ms}$ ). A2. Plot shows the rebound sag amplitude (ordinate) measured in a $\mathrm{pv}+\mathrm{BC}$ at $-70 \mathrm{mV}$ following membrane potential steps (Vm, see insets) in the control conditions (blue symbols) and in the presence of ZD (black). The rebound sag amplitude gives a reliable measure that allows to compare the strength of $\mathrm{HCN}$-channel mediated responses at different somatic membrane potentials, with the sag amplitude systematically measured at $-70 \mathrm{mV}$ to prevent any variable electrochemical driving force. Insets: Specimen membrane potential traces of a depolarizing or 
hyperpolarizing step in control (blue) and in ZD (black) conditions. HCN rebound sag amplitude is defined in traces with dotted lines and a bracket (scale $10 \mathrm{mV}, 100 \mathrm{~ms}$ ). A3. The rebound $\mathrm{HCN}$ sag amplitude measured in similar way in 11 human pv+BCs following depolarizing voltage steps (from $-70 \mathrm{mV}$ to $-50 \mathrm{mV}$ ) and hyperpolarizing steps (to $-90 \mathrm{mV}$ from $-70 \mathrm{mV})$. Blue shows control and black the $\mathrm{ZD}(10 \mu \mathrm{M})$ conditions $(\mathrm{P}<0.001$ and $\mathrm{P}<$ 0.001, Wilcoxon Signed-Rank Test).

B. Larger somatic HCN sag potential observed in human compared to mouse pv+BCs. B1. The cumulative histogram shows $\mathrm{HCN}$ sag potential amplitude (as illustrated in one cell in inset) evoked by hyperpolarizing somatic step from $-70 \mathrm{mV}$ to $-90 \mathrm{mV}$ in identified pv+BCs. The HCN sag amplitude is larger in human cells (blue, $n=28$ ) compared to mouse cells (green, $\mathrm{n}=32)(\mathrm{P}<0.001$, Mann-Whitney U-test). Insets: Traces illustrate robust $\mathrm{HCN}$ voltage sag in a human but not in a mouse pv+BC (scale $50 \mathrm{~ms}$ ). B2. Cumulative histogram of $\mathrm{HCN}$ sag amplitude in human and mouse pv+BCs by membrane potential steps from $-50 \mathrm{mV}$ to $-70 \mathrm{mV}$. The $\mathrm{HCN}$ sag amplitude in human is larger than in mouse $(\mathrm{P}<0.001$, MannWhitney U-test). Insets: Sample traces in a human and in a mouse pv+BCs (scale $10 \mathrm{mV}, 50$ $\mathrm{ms})$.

C. A robust $\mathrm{HCN}$ sag occurs in the human pv+BCs among the superficial or supragranular L2/3. C1. Illustration of one human $p v+B C$ in $L 2 / 3$. Confocal imaging micrographs show positive immunofluorescence reaction of the biocytin-filled (bioc) neuron to parvalbumin (pv) with a soma location at superficial L2. Left: Fluorescence micrographs illustrate biocytin (bioc)-filled soma immunopositive for parvalbumin (pv). Scale: $10 \mu \mathrm{m}$. Partial anatomical reconstruction shows cell soma and dendrites (red), as well as axon (black) in two $60 \mu \mathrm{m}$ thick merged sections. Scale: $100 \mu \mathrm{m}$. Horizontal lines shows L1 and L4 borders. C2. Abscissa summarizes $\mathrm{HCN}$ sag amplitude in human $\mathrm{pv}+\mathrm{BC}$ and ordinate shows the soma location in 
$\mathrm{L} 2 / 3$ relative to $\mathrm{L} 1$ border (indicated as $0 \%$ ) and to $\mathrm{L} 4$ border (indicated as $100 \%$ in the plot). Percentage bin is $5 \%$. Red dot in the plot belongs to the sample cell shown on the right in full scale (scale $100 \mu \mathrm{m}$ ). Sag values are from 27 cells in $B 1$ data with a successful anatomical localization of the soma.

Figure 2. HCN-channel activity contributes to determining human pv+BC resting membrane potential

A1. Symbols show the resting membrane potential $(\mathrm{Em})$ in 18 human and 18 mouse pv+BCs (blue and green, respectively) (Em between the species $P=0.080$, Student's t-test) (human $-66.33 \pm 7.15 \mathrm{mV}$, mean \pm standard deviation (SD), $\mathrm{n}=18$; and mouse $-61.17 \pm 9.84 \mathrm{mV}, \mathrm{n}=$ 18). A2. HCN sag amplitude in the pv+BCs measured at Em following a depolarizing step to $-50 \mathrm{mV}$. The robust depolarizing pulse aims to inactivate the $\mathrm{HCN}$ channels, which reactivate at Em by the repolarization as illustrated in the inset.

Bar histogram shows $\mathrm{HCN}$ sag amplitude from the largest to the smallest in human and mouse pv+BCs. Individual cells are identified by codes in abscissa.

B1. Cumulative histogram collates $\mathrm{HCN}$ sag amplitudes at Em in the human and mouse pv+BCs. The sag amplitude is larger in human than in mouse pv+BCs $(P<0.001$, MannWhitney U-test). B2. Plot summarizes the Em values (Em) and $\mathrm{Vm}$ values in the cells when a depolarizing effect of the sag amplitude (when applicable) is subtracted (E-sag). B3. Dataset from small number of separate experiments show that the ZD wash-in generates similar negative shift of Em in human $(n=11)$ but not in mouse $(n=7)$ pv+BCs (paired Student's ttest). 


\section{Figure 3. HCN channels maintain somatic leak conductance in human pv+BCs at the resting}

\section{membrane potential}

A. Human pv+ interneurons in L2/3 show strong immunopositivity for hyperpolarizationactivated cyclic nucleotide-gated channel 1 (HCN1). Confocal images showing co-localization of pv and HCN1 immunofluorescence in L2/3. Micrographs Ai-iii are different neocortical samples. Alexa488 (green) and cy3 (red) (m, merge). Scale $10 \mu \mathrm{m}$.

B. Voltage-clamp step shows a leak conductance reduction in human $\mathrm{pv}+\mathrm{BC}$ with application of the HCN-channel blocker ZD7288 (ZD; $10 \mu \mathrm{M})$. B1. Sample traces (averages of five) show somatic voltage-clamp current for -10 and $+10 \mathrm{mV}$ sequential voltage steps (10 ms) measured at Em. Blue trace is in control conditions and black trace in the presence of ZD (10 $\mu \mathrm{M})$. Voltage-clamping $\mathrm{pv}+\mathrm{BC}$ at $\mathrm{Em}(-65 \mathrm{mV}$ throughout the experiment) was associated with a moderate $(+12 \mathrm{pA})$ shift of holding current level in ZD. Scale $100 \mathrm{pA}, 50 \mathrm{~ms}$. B2. Zoomed-in and superimposed traces (averages of five, the holding current levels are aligned for clarity) illustrate the voltage-clamp current to a $-10 \mathrm{mV}$ step in the two conditions.

There is a decrease in current amplitude for the voltage step in ZD as indicated by brackets; Current step amplitude in control and in ZD are marked as $I_{c t r l}$ and $I_{Z D}$ respectively.

$I_{\text {ctrl }}$ or $I_{\text {ZD }}$ amplitude is proportional to somatic leak conductance. Scale 100 pA, 10 ms. B3. Plot shows the current amplitude for $-10 \mathrm{mV}$ (solid symbols) and $+10 \mathrm{mV}$ (open symbols) voltage steps before and during the wash-in with ZD (indicated with gray background) in the same experiment.

C. Voltage-clamp step current is not changed in a mouse pv+BC by ZD. C1-3. ZD wash-in shows negligible effect in identical experiment conducted in a mouse pv+BC. Scales: 200 pA, $50 \mathrm{~ms}$ and $100 \mathrm{pA}, 10 \mathrm{~ms}$. 
D. Plot compiles leak conductance $(\mathrm{nS})$ values translated from the voltage-clamp step current in 5 human and 5 mouse pv+BCs in control conditions (baseline, bl) and in ZD. ZDsensitive conductance $\left(\mathrm{G}_{\mathrm{HCN}}\right)$ was $11.9 \%, 1.8 \%-30 \%$ of total leak conductance in human, and 4.36\%, 2.7\%-5.4\% in mouse cells ( $P=0.151$ between the human and mouse, MannWhitney U-test).

Figure 4. HCN-channel activity shortens the somatic time-constant and the EPSP time-topeak in human pv+BCs

A. HCN-channel activity keeps human pv+BC membrane tau close to tau in mouse cells. $A 1$. Plot shows membrane time constant (membrane tau) measured in human pv+BC soma during different membrane potential steps delivered at $-70 \mathrm{mV}$ in control conditions (blue) and in ZD7288 (ZD; black). Inset: traces illustrate membrane potential step (average of five traces) from $-70 \mathrm{mV}$ to $-90 \mathrm{mV}$ in control (blue) and in ZD (black). Scale $10 \mathrm{mV}, 5 \mathrm{~ms}$. Istep indicates the hyperpolarizing current step. A2. Cumulative histograms show passive membrane time constant (membrane tau) measured during $V m$ steps from $-70 \mathrm{mV}$ to -90 $m V$ in human (blue, $n=8$ ) and mouse (green, $n=7$ ) pv+BCs. Solid line is in control conditions and dotted line is in the presence of ZD. ZD significantly prolongs the passive membrane tau both in human $(n=8, P=0.008)$ and in mouse $(n=7, P=0.031) p v+B C s$. Yet in the presence of $Z D$, human $p v+B C$ tau (dotted blue line) is significantly different from mouse $\mathrm{pv}+\mathrm{BC}$ values (green lines), whereas in control condition there is no significant difference between the human and mouse tau values (Kruskal-Wallis H-test and multiple comparisons against control group with Dunn's method). A3. ZD effect on passive membrane tau is strongest in pv+BC showing the largest $\mathrm{HCN}$ sag potential amplitude in control conditions. Plot shows the baseline-normalized membrane tau value in ZD (ordinate) 
against the pv+BC HCN sag amplitude (abscissa) measured in cells under control conditions. Pearson correlation indicates linear correlation (Coefficient $=0.905, P>0.001, n=15$ cells, $\left.r^{2}=0.818\right)$. Dotted line shows regression. The sag and the tau were measured in Vm steps from $-70 \mathrm{mV}$ to $-90 \mathrm{mV}$.

B. ZD prolongs somatic EPSPs time-to-peak (applied with dynamic-clamp) in human pv+BCs. B1. Plot shows time-to-peak (ordinate) for different EPSP amplitudes (abscissa) in a sample human pv+BC in control conditions (blue) and in ZD (black). EPSPs were generated with incremental EPSCs strength (EPSCs not shown) at $-70 \mathrm{mV}$. Inset: EPSP of similar amplitude in control and in ZD (average of five) illustrate longer time-to-peak (indicated with brackets) in the presence of ZD. Scale: $5 \mathrm{mV}, 2 \mathrm{~ms}$. B2. ZD effect on EPSP time-to-peak (ttp) tested in randomly selected 9 human (blue) and 8 mouse (green) pv+BCs. EPSPs were evoked by dynamic-clamp and values ttp-values are measured from EPSPS of similar amplitude in control and ZD (Human $\mathrm{P}=0.004$; mouse $\mathrm{P}=0.029$, paired t-test) (Shapiro-Wilk normality $\mathrm{P}$ $=0.546$ and $P=0.705$, respectively). EPSP ttp increases significantly both in human and in mouse cells. However, effect of ZD on EPSP time-to-peak is stronger in the human than in mouse pv+BCs $(P=0.049$, t-test. Shapiro-Wilk normality $P=0.34)$. Baseline-normalized EPSP amplitude (R) in ZD was $1.18 \pm 0.14$ in human and $1.07 \pm 0.06$ in mouse cells. B3. Cumulative histogram shows the EPSP time-to-peak values in control and in ZD. EPSP ttp is longer in human than in mouse both in control conditions and in ZD. (Control, solid line; ZD dotted line). One-way ANOVA ( $\mathrm{P}<0.001)$ with pairwise multiple comparison (Holm-Sidak method).

Figure 5. HCN-channel activity shortens EPSC-to-spike delay in human pv+BCs 
A. Dot plot shows EPSC-to-spike delay (abscissa) in control and in the presence of ZD in one human pv+BC. Four different EPSC strength were used in both conditions to elicit EPSP-spike compound with four different spiking probability (ordinate). EPSCs are not identical strength in control and in ZD, but their strength was adjusted to aim EPSPs with similar spike probability in the two conditions. Blue and black symbols show delay of the spikes evoked when 30 cycles by each EPSC strength was tested (interval $1 \mathrm{~s}$ ). Membrane potential is -70 $\mathrm{mV}$ throughout the experiment. $P$ values (paired Student's t-test) are tested for delay values when spikes were elicited with similar probability in control (blue) and in ZD (black) (control 0.56 and in ZD 0.53; control 0.98 and in ZD 0.94). Inset: traces illustrate EPSP with spike (blue) elicited with EPSC (gray, dynamic-clamp) at $-70 \mathrm{mV}$. Dotted lines and the bracket illustrate EPSC-to-spike delay, with traces showing dynamic-clamp EPSC (gray) and the EPSP with spike. Scale: 200 pA, $25 \mathrm{mV}, 2 \mathrm{~ms}$.

B. ZD increases EPSC-to-spike delay in various individual human pv+BCs. B1. Superimposed EPSP-spike traces in one human pv+BC (same cell as in A) in control conditions (blue) and in ZD (black). Traces show EPSP-spike compounds with spike probability of 0.5 and close to 1. Histograms summarize EPSC-to-spike delay illustrated with the traces. B2. Cumulative histograms show EPSC-to-spike delay in 3 human pv+BCs and in 1 mouse pv+BC under control (blue, green) and ZD (black) conditions. Spike probability value ( $\mathrm{pr}$ for 30 EPSC cycles) is indicated in the plots.

C. ZD effect on EPSC-to-spike delay is largest in pv+BCs showing the strongest effect of ZD on the passive membrane time-constant (measured during the hyperpolarizing step). Pearson correlation indicates linear correlation (Coefficient $=0.619, P=0.0129, n=11$ cells, $\left.r^{2}=0.383\right)$. Blue, human $p v+B C s$, green, mouse pv+BCs. Dotted line shows regression. 
Figure 6. Somatic $\mathrm{HCN}$ conductance facilitates $\mathbf{p v + B C}$ input-to-output function in a computational model

$\mathrm{HCN}$ channel-mediated leaking conductance $\left(\mathrm{G}_{\mathrm{HCN}}\right)$ in the human pv+BCs attenuates somatic EPSP amplitude. However, a large depolarizing effect of $\mathrm{G}_{\mathrm{HCN}}$ on the resting membrane potential (Em) dominates the EPSP amplitude attenuation. The net effect by $\mathrm{G}_{\mathrm{HCN}}$ is that EPSCS generate EPSPS reaching higher depolarizing level and with shorter delay.

A. Computational single-cell passive membrane model replicates human pv+BCs with authentic cell capacitance, $\mathrm{Em}, \mathrm{G}_{\mathrm{HCN}}$, and ZD-insensitive leak conductance measured in the cells. The analysis includes 5 human and 5 mouse $\mathrm{pv}+\mathrm{BC}$ studied in Figure 3. A1-3. Illustration of three human pv+BC simulations. Traces show EPSPS evoked in the soma by same EPSC (3.4-5.0 nS) at resting membrane potential (Em) in control conditions (blue) or without the ZD-sensitive $\mathrm{G}_{\mathrm{HCN}}(\mathrm{Erev}=-30 \mathrm{mV}$ ). Bar charts show the relative proportion of $\mathrm{G}_{\mathrm{HCN}}$ (white area) and the ZD-insensitive conductance (g no HCN, black area) of the total leak conductance (blue bar) measured in the cell in control conditions. In the absence of $\mathrm{G}_{\mathrm{HCN}}$, Em is hyperpolarized (black font). Horizontal dotted line shows a reference line to compare the membrane potential depolarization level reached by EPSPS at specific time point. Shaded gray area highlights the time delay between the EPSPS to reach a nominal depolarization level.

$B$. Simulation experiments illustrated in three mouse cells (B1-B3) shows that the modest somatic $\mathrm{G}_{\mathrm{HCN}}$ in these cells has negligible effect on somatic Em or the EPSPS.

C. Modeling data demonstrate that net effect of somatic $\mathrm{G}_{\mathrm{HCN}}$ in pv+BCs is their facilitated excitation. C1. Bar histogram shows the ratio of $\mathrm{G}_{\mathrm{HCN}}$ to the total leak conductance (Gtotal) measured in the 5 human (blue) and 5 mouse (green) pv+BCs at Em. Abscissa shows the cell codes. C2. Same cells as in C1 showing the EPSP amplitude (vertical bar) at Em (horizontal 
line at bottom of the bars) in control conditions (with $\mathrm{G}_{\mathrm{HCN}}$, blue or green) and in the absence of $\mathrm{G}_{\mathrm{HCN}}$ (black). EPSC underlying the EPSPs differs in strength between the cells, and it was adjusted to elicit an EPSP that reaches the average firing threshold level (indicated by horizontal dotted line) in the control conditions. The bars show that with $\mathrm{G}_{\mathrm{HCN}}$ in cell soma, the Em depolarizes more than the EPSP amplitude attenuates. When $\mathrm{G}_{\mathrm{HCN}}$ is off, the EPSPS (black bars) fail to reach the firing threshold level from the hyperpolarized Em. C3. Summary of the $\mathrm{GHCN}_{\mathrm{H}}$ effect on EPSP amplitude and Em in the pv+BCs. Vertical bars demonstrate the increase of EPSP amplitude (upper bars) and the negative shift of Em (lower bars) in the absence of $G_{H C N}$ in the cells. The shift in Em is larger than the increase in EPSP amplitude ( $n$ $=10, P=0.002$, Wilcoxon Signed-Rank Test).

D. Zoomed-in traces showing the shaded area between EPSPS in the 3 human pv+BCs illustrated in A1-3. Depolarization by EPSPS is slower in the absence of $\mathrm{G}_{\mathrm{HCN}}$ (black).

\section{Supplementary Figure S1. HCN potential alone does not explain Em variation in pv+BCs}

$\mathrm{HCN}$ sag potential amplitude (measured at Em) does not show correlation with the Em value in either human (correlation coefficient $=0.314, \mathrm{P}=0.198$ ) or mouse $(0.416$ and 0.084 , respectively) pv+BCs (spearman Rank order correlation). Dotted line indicates regression.

\section{Supplementary Figure $S 2$. Blocking $\mathbf{G}_{\mathrm{HCN}}$ increases somatic EPSP amplitude}

A. Dynamic-clamp EPSC (gray trace) in a sample recording from human $\mathrm{pv}+\mathrm{BC}$ demonstrates increased EPSP amplitude in the presence of ZD (black trace) compared to baseline control (blue trace).

B. The plot shows that the increase in amplitude correlates with an increase of the somatic passive input resistance $\mathrm{Rm}$ (measured from a hyperpolarizing $-20 \mathrm{mV}$ step at $-70 \mathrm{mV}$ in 
current clamp) $\left(P=0.037\right.$, Spearman Rank Order Correlation. $\left.r^{2}=0.327\right)$. The EPSP amplitude increase by ZD effect is smaller than expected from the changes in passive membrane properties, probably because robust depolarizing EPSPs close to firing threshold are additionally associated with voltage-sensitive conductances. Data include 6 human and 4 mouse pv+BCs.

Supplementary Figure S3. Spiking probability evoked by equal-amplitude EPSP is not significantly different between baseline control and ZD

Spiking probability evoked by equal-amplitude EPSPs (dynamic-clamp) delivered from -70 $\mathrm{mV}$ in baseline control (open symbols) and in the presence of ZD (solid symbols) (human, left; mouse, right). Probability of spiking (P), evoked by EPSPs applied at $-70 \mathrm{mV}$ in baseline and following wash-in of ZD is not changed (Wilcoxon Signed-Rank Test $P=0.688, n=7$ ).

Supplementary Table 1. List of anatomically identified and pv+ immunopositive human basket cells, and anatomically identified mouse fast-spiking basket cells of the study. Sheets show codes of the experiments included in specific datasets and figures.

Supplementary Table 2. A Voltage sag ('sag during') and a rebound sag ('rebound sag at Em) amplitude in individual experiments (identified by cell code). 'Step from Em' shows the hyperpolarizing step peak amplitude (step delivered at -70 mV).

Supplementary Table 3. Table shows intrinsic pvBC parameters used for modelling in Figure 6. 
METHODS

Ethics statement. All the procedures were performed according to the Declaration of Helsinki with the approval of the University of Szeged Ethical Committee and the Regional Human Investigation Review Board (ref. 75/2014). For all human tissue material, written consent was obtained from patients prior to surgery. Tissue obtained from minor patients was provided with agreement from a parent or legal guardian.

Human brain slices. Neocortical slices were sectioned from material that had to be removed to gain access for the surgical treatment of deep-brain targets from the frontal, temporal, or occipital areas. The patients were 14-74 years of age, and samples from males and females from either the left or right hemisphere were included. Anesthesia was induced with intravenous midazolam and fentanyl $(0.03 \mathrm{mg} / \mathrm{kg}, 1-2 \mathrm{lg} / \mathrm{kg}$, respectively). A bolus dose of propofol (1-2 mg/kg) was administered intravenously. The patients also received $0.5 \mathrm{mg} / \mathrm{kg}$ rocuronium to facilitate endotracheal intubation. The trachea was intubated, and patients were ventilated with an $\mathrm{O}_{2} / \mathrm{N}_{2} \mathrm{O}$ mixture at a ratio of $1: 2$. Anesthesia was maintained with sevoflurane. Following surgery, the resected tissue blocks were immediately immersed into ice-cold solution within a glass inside the operating room. The solution contained (in $\mathrm{mM}$ ): $130 \mathrm{NaCl}, 3.5 \mathrm{KCl}, 1 \mathrm{NaH}_{2} \mathrm{PO}_{4}, 24 \mathrm{NaHCO}_{3}, 1 \mathrm{CaCl}_{2}, 3 \mathrm{MgSO}_{4}, 10 \mathrm{D}(+)$-glucose and was saturated with $95 \% \mathrm{O}_{2} / 5 \% \mathrm{CO}_{2}$. The container was placed on ice in a thermally isolated transportation box where the liquid was continuously gassed with $95 \% \mathrm{O}_{2} / 5 \% \mathrm{CO}_{2}$. Then, the tissue was immediately transported from the operating room to the electrophysiology laboratory where slices of $350 \mu \mathrm{m}$ thickness were prepared from the tissue block with a 
vibrating blade microtome (Microm HM 650 V). The slices were incubated at $22^{\circ} \mathrm{C}-24^{\circ} \mathrm{C}$ for $1 \mathrm{~h}$, when the slicing solution was gradually replaced by the solution used for storage (180 $\mathrm{ml})$ using a pump $(6 \mathrm{ml} / \mathrm{min})$. The storage solution was identical to the slicing solution, except that it contained $3 \mathrm{mM} \mathrm{CaCl}_{2}$ and $1.5 \mathrm{mM} \mathrm{MgSO}_{4}$.

Drug. ZD7288 (Sigma Aldrich, Budapest, Hungary) was diluted in physiological extracellular solution and applied by wash-in.

Identification of fast-spiking BCs in mouse brain slices. Transversal slices (350 $\mu \mathrm{m})$ from the somatosensory and in some cases the frontal cortex were prepared from 4- to 6-week-old heterozygous B6.129P2-Pvalbtm1(cre)Arbr/J mice (The Jackson Laboratory, stock 017320, B6 PVcre line) expressing td-Tomato fluorophore preferentially in parvalbumin GABAergic neurons. Cells were confirmed to be fast-spiking by electrophysiological recording of fast spike kinetics and high-frequency non-accommodating firing pattern to suprathreshold depolarizing $500 \mathrm{~ms}$ pulses. Cells were visualized with streptavidin Alexa488 (1:2000, Jackson ImmunoResearch Lab, Inc.) and identified visually under epifluorescence microscopy.

Electrophysiology. Recordings were performed in a submerged chamber (perfused 8 $\mathrm{ml} / \mathrm{min}$ ) at $36^{\circ} \mathrm{C}-37^{\circ} \mathrm{C}$. Cells were patched using a water-immersion $20 \mathrm{x}$ objective with additional zoom (up to $4 \mathrm{x}$ ) and infrared differential interference contrast video microscopy. Micropipettes (5-8 M $\Omega$ ) for whole-cell patch-clamp recording were filled with intracellular solution (in mM): 126 K-gluconate, $8 \mathrm{NaCl}, 4$ ATP-Mg, $0.3 \mathrm{Na}_{2}-\mathrm{GTP}, 10$ HEPES, and 10 phosphocreatine ( $\mathrm{pH} 7.0-7.2 ; 300 \mathrm{mOsm})$ with $0.3 \%(\mathrm{w} / \mathrm{v})$ biocytin. Recordings were 
performed with a Multiclamp 700B amplifier (Axon Instruments) and low-pass filtered at a 6-8 kHz cut-off frequency (Bessel filter). Series resistance and pipette capacitance were compensated in current-clamp mode and pipette capacitance was compensated in voltageclamp mode. Liquid junction potential error was not corrected in membrane potential values. The access resistance of the recording electrode was measured, and its effect on the clamping potential error was corrected in nominal somatic potential reading. Em was recorded 1-3 min after break-in to whole cell. Cell capacitance was measured in current clamp using -50-100 pA, 200-300 ms steps delivered at Em. The somatic leaking conductance in Figure 3 was measured in voltage clamp at Em by delivering $-10 \mathrm{mV}$ and 10 $\mathrm{mV}(10 \mathrm{~ms})$ voltage steps, and conductance was calculated from clamping current amplitude as demonstrated in Figure 3 using the Ohm's equation. Somatic input resistance in current clamp was measured from hyperpolarizing $(-10$ to $-20 \mathrm{mV}, 250 \mathrm{~ms})$ voltage steps at -70 $\mathrm{mV}$.

Dynamic clamp. To induce EPSPS, a software-based dynamic-clamp system was employed. Current injections were calculated and delivered by the Signal software (Cambridge Electronic Design, Cambridge, UK) through a Power1401-3A data acquisition interface (CED, Cambridge, UK) based on voltage signals of the electrode. We ran the dynamic clamp on a computer distinct from our experimental data acquisition system (recording cell membrane potential) to record and verify dynamic-clamp output (conductance and EPSCS). EPSCs were generated using a decay time constant of $3 \mathrm{~ms}$ and a reversal potential of $0 \mathrm{mV}$. The peak conductance (1.5-10 nS) was set to evoke EPSPs. 
Single-cell model. For the simulation of somatic EPSP, real experimental data from individual basket cells were used. Simulation of a basket cell membrane potential was performed using a NEURON 7.6.5 simulator (Carnevale NT HMC, UK: Cambridge University Press 2006). The membrane capacitance was $1 \mu \mathrm{F} / \mathrm{cm}^{2}$, and the size of the soma was determined so that the total cell capacitance matched the actual measured value in each cell. $G_{\text {leak }}$ (total $G$ at resting $\mathrm{Em}$ ) and $\mathrm{G}_{\mathrm{HCN}}(\mathrm{ZD}$-sensitive conductance) were retrieved from voltage-clamp data in Figure 3 human and mouse identified neurons. $G_{H C N}$ reversal potential was $-30 \mathrm{mV}$, and $G_{\text {leak }}$ reversal potential was set at the resting membrane potential value measured in the presence of ZD in each recorded cell to reach the actual Em value measured in control conditions. EPSPS were modeled using a rise tau of $0.2 \mathrm{~ms}$, decay tau of $3 \mathrm{~ms}$ and conductance of $10 \mathrm{nS}$ with a reversal potential of $0 \mathrm{mV}^{12,14}$.

Data analysis. Data were acquired using the Clampex software (Axon Instruments) and digitized at $35-50 \mathrm{kHz}$. The data were analyzed off-line with pClamp (Axon Instruments), Spike2 (version 8.1, Cambridge Electronic Design), OriginPro (OriginLab Corporation) and SigmaPlot14 software (Systat Software).

Statistics. Data are presented as the mean $\pm S D$, for samples with size $(n) \geq 7$ and a parametric distribution. Normality was tested with the Shapiro-Wilk test using a $\mathrm{P}$ value $>$ 0.05. Otherwise, data are shown as the median with interquartile range (of lower and upper quartile) or as the average and range, unless stated otherwise. Correspondingly, for statistical analysis, Student's two-tailed or paired t-test, Mann-Whitney U-test, Wilcoxon Signed-Rank Test or two-tailed ANOVA or Kruskal-Wallis H-test (with Dunn's post-hoc test) 
were used with Sigma Plot. Correlations were tested using Pearson or Spearman correlation, respectively. Differences were considered significant at $P<0.05$.

Tissue fixation and cell visualization. Biocytin-filled cells were visualized by using either Alexa488- (1:500) or Cy3-streptavidin (1:400, Jackson ImmunoResearch Lab, Inc.). After electrophysiological recording, slices were immediately fixed in a fixative solution containing $4 \%$ paraformaldehyde and $15 \%$ picric acid in $0.1 \mathrm{M}$ phosphate buffer $(\mathrm{PB}, \mathrm{pH}=7.4)$ at $4^{\circ} \mathrm{C}$ for at least $12 \mathrm{~h}$ and then stored at $4^{\circ} \mathrm{C}$ in $0.1 \mathrm{M} \mathrm{PB}$ containing $0.05 \%$ sodium azide as a preservative. All slices were embedded in $10 \%$ gelatin and further sectioned into slices of $60 \mu \mathrm{m}$ thickness in ice-cold PB using a vibratome VT1000S (Leica Microsystems). After sectioning, the slices were rinsed in $0.1 \mathrm{M} \mathrm{PB}(3 \times 10 \mathrm{~min})$ and cryoprotected in $10-20 \%$ sucrose solution in 0.1? ? M PB. Afterwards, the slices were flash-frozen in liquid nitrogen and thawed in 0.1 M PB. Finally, they were incubated in a fluorophore-conjugated streptavidin (1:400 or 1:500, Jackson ImmunoResearch Lab, Inc.) in $0.1 \mathrm{M}$ Tris-buffered saline (TBS, pH 7.4) for $2.5 \mathrm{~h}$ (at $\left.22^{\circ} \mathrm{C}-24^{\circ} \mathrm{C}\right)$. After washing with $0.1 \mathrm{M} \mathrm{PB}(3 \times 10 \mathrm{~min})$, the sections were covered in Vectashield mounting medium (Vector Laboratories Inc.), placed under coverslips, and examined under an epifluorescence microscope with 20-60 x magnification (Leica DM 5000 B).

Cell reconstruction and anatomical analyses. Sections used for morphological analyses in Figure $1 \mathrm{C}$ were further incubated in a solution of conjugated avidin-biotin horseradish peroxidase ( $A B C$; $1: 300$; Vector Labs) in TBS $(\mathrm{pH}=7.4)$ at $4^{\circ} \mathrm{C}$ overnight. The enzyme reaction was revealed with the glucose oxidase-DAB-nickel method using 3?3diaminobenzidine tetrahydrochloride $(0.05 \%)$ as the chromogen and $0.01 \% \mathrm{H}_{2} \mathrm{O}_{2}$ as the 
oxidant. Sections were further treated with $1 \% \mathrm{OsO}_{4}$ in $0.1 \mathrm{M}$ PB. After several washes in distilled water, sections were stained in $1 \%$ uranyl acetate and dehydrated in an ascending series of ethanol concentrations. Sections were infiltrated with epoxy resin (Durcupan) overnight and embedded on glass slides. For the cell shown in the Figure 1 threedimensional light microscopic reconstructions from two sections were carried out using the Neurolucida system with $100 x$ objective (Olympus BX51, Olympus UPlanFI). Images were collapsed in the z-axis for illustration. For laminar depth analysis shown in Figure 1, the soma location and the borders of L1/L2 and L3/L4 were defined in individual $60 \mu \mathrm{m}$-thick sections using a brightfield microscope following the conversion to DAB.

Immunohistochemistry. Free-floating sections were washed 3 times in TBS-TritonX $0.3 \%$ (15 $\min$ ) at $22^{\circ} \mathrm{C}-24^{\circ} \mathrm{C}$ and then transferred to $20 \%$ blocking solution with horse serum in TBSTritonX, $0.3 \%$ for parvalbumin (pv) staining. For some sections, pre-treatment with pepsin was performed to improve immunohistochemical staining. The sections were treated with 1 $\mathrm{mg} / \mathrm{ml}$ pepsin (catalog \#S3002; Dako) in $1 \mathrm{M} \mathrm{HCl}$ with $0.1 \mathrm{M} \mathrm{PB}$ at $37^{\circ} \mathrm{C}$ for 6 min and then washed in $0.1 \mathrm{M} \mathrm{PB}$. All sections were incubated in primary antibodies diluted in $1 \%$ serum in TBS-TritoX $0.3 \%$ over three nights at $4^{\circ} \mathrm{C}$, and then placed in relevant fluorochromeconjugated secondary antibody solutions (1 \% blocking serum in TBS-TritonX $0.3 \%$ ) overnight at $4^{\circ} \mathrm{C}$. Sections were first washed in TBS-TritonX $0.3 \%(3 \times 20 \mathrm{~min})$ and later in $0.1 \mathrm{M}$ PB $(3 \times 20 \mathrm{~min})$ and mounted on glass slides with Vectashield mounting medium (Vector Lab, Inc.). The characterizations of primary antibodies used in humans: mouse antipv (1:500, Swant, Switzerland, www.swant.com, clone: 235); rabbit anti-HCN1 1: 500, MyBioSource). Fluorophore-labeled secondary antibodies were (DAM DyLight 488 donkey anti-mouse, 1:400, Jackson ImmunoResearch Lab. Inc., www.jacksonimmuno.com) or (DAM 
Cy3 donkey anti-mouse, 1:400, Jackson ImmunoResearch Lab. Inc.,

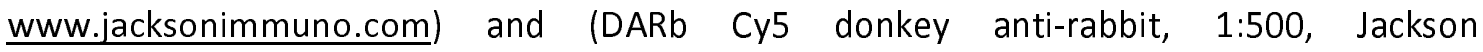
ImmunoResearch Lab. Inc.). The immunoreactions were evaluated using first epifluorescence (Leica DM 5000 B) and then laser scanning confocal microscopy (Zeiss LSM880). All the micrographs presented are confocal fluorescence microscopy images.

\section{ACKNOWLEDGEMENTS}

This work was supported by OTKA K 134279 (VS, SF, KL), the National Brain Research Program Hungary (VS, GT and KL), the European Research Council INTERIMPACT project (GT), the Hungarian Academy of Sciences (GT), the National Research, Development and Innovation Office of Hungary (GINOP-2.3.2-15-2016-00018, VKSZ-14-1-2015-0155, GT), the Ministry of Human Capacities, Hungary (grant 20391-3/2018/FEKUSTRAT, GT) and by University of Szeged Open Access Fund (Grant number: 4373). We acknowledge Ms Leona Mezei and Drs Gábor Molnár and Katalin Kocsis for technical assistance. 
1. $\mathrm{Hu} \mathrm{H}$, Gan J, Jonas P. Interneurons. Fast-spiking, parvalbumin(+) GABAergic interneurons: from cellular design to microcircuit function. Science 345, 1255263 (2014).

2. Wood KC, Blackwell JM, Geffen MN. Cortical inhibitory interneurons control sensory processing. Curr Opin Neurobiol 46, 200-207 (2017).

3. Jonas P, Bischofberger J, Fricker D, Miles R. Interneuron Diversity series: Fast in, fast out--temporal and spatial signal processing in hippocampal interneurons. Trends Neurosci 27, 30-40 (2004).

4. Fricker D, Miles R. EPSP amplification and the precision of spike timing in hippocampal neurons. Neuron 28, 559-569 (2000).

5. Zsiros V, Hestrin S. Background synaptic conductance and precision of EPSP-spike coupling at pyramidal cells. J Neurophysiol 93, 3248-3256 (2005).

6. $\quad \mathrm{Li}$ T, et al. Action potential initiation in neocortical inhibitory interneurons. PLoS Biol 12, e1001944 (2014).

7. Debanne D, Russier M. The contribution of ion channels in input-output plasticity. Neurobiol Learn Mem 166, 107095 (2019).

8. Olah S, et al. Output of neurogliaform cells to various neuron types in the human and rat cerebral cortex. Front Neural Circuits 1, 4 (2007).

9. Verhoog MB, et al. Mechanisms underlying the rules for associative plasticity at adult human neocortical synapses. J Neurosci 33, 17197-17208 (2013).

10. Molnar G, et al. Complex events initiated by individual spikes in the human cerebral cortex. PLoS Biol 6, e222 (2008).

11. Komlosi G, Molnar G, Rozsa M, Olah S, Barzo P, Tamas G. Fluoxetine (prozac) and serotonin act on excitatory synaptic transmission to suppress single layer $2 / 3$ pyramidal neuron-triggered cell assemblies in the human prefrontal cortex. J Neurosci 32, 1636916378 (2012).

12. Szegedi V, et al. High-Precision Fast-Spiking Basket Cell Discharges during Complex Events in the Human Neocortex. eNeuro 4, (2017).

13. Chittajallu R, et al. Activity-dependent tuning of intrinsic excitability in mouse and human neurogliaform cells. Elife 9, (2020).

14. Molnar $G$, et al. Human pyramidal to interneuron synapses are mediated by multivesicular release and multiple docked vesicles. Elife 5, (2016). 
15. Szegedi V, et al. Plasticity in Single Axon Glutamatergic Connection to GABAergic Interneurons Regulates Complex Events in the Human Neocortex. PLoS Biol 14, e2000237 (2016).

16. Testa-Silva $\mathrm{G}$, et al. High bandwidth synaptic communication and frequency tracking in human neocortex. PLoS Biol 12, e1002007 (2014).

17. Eyal $\mathrm{G}$, et al. Unique membrane properties and enhanced signal processing in human neocortical neurons. Elife 5, (2016).

18. Kalmbach BE, et al. h-Channels Contribute to Divergent Intrinsic Membrane Properties of Supragranular Pyramidal Neurons in Human versus Mouse Cerebral Cortex. Neuron 100, 1194-1208 e1195 (2018).

19. Poorthuis RB, et al. Rapid Neuromodulation of Layer 1 Interneurons in Human Neocortex. Cell Rep 23, 951-958 (2018).

20. Warming $\mathrm{H}$, et al. A primate-specific short GluN2A-NMDA receptor isoform is expressed in the human brain. Mol Brain 12, 64 (2019).

21. Luke Campagnola SCS, Thomas Chartrand, Lisa Kim, Alex Hoggarth, et al. Connectivity and Synaptic Physiology in the Mouse and Human Neocortex. bioRxiv, (2021).

22. Defelipe J. The evolution of the brain, the human nature of cortical circuits, and intellectual creativity. Front Neuroanat 5, 29 (2011).

23. Katz PS, Harris-Warrick RM. The evolution of neuronal circuits underlying speciesspecific behavior. Curr Opin Neurobiol 9, 628-633 (1999).

24. Zecevic N, Hu F, Jakovcevski I. Interneurons in the developing human neocortex. Dev Neurobiol 71, 18-33 (2011).

25. Hodge RD, et al. Conserved cell types with divergent features in human versus mouse cortex. Nature 573, 61-68 (2019).

26. Blazquez-Llorca L, Garcia-Marin V, DeFelipe J. GABAergic complex basket formations in the human neocortex. J Comp Neurol 518, 4917-4937 (2010).

27. Hu X, Rocco BR, Fee C, Sibille E. Cell Type-Specific Gene Expression of Alpha 5 Subunit-Containing Gamma-Aminobutyric Acid Subtype A Receptors in Human and Mouse Frontal Cortex. Mol Neuropsychiatry 4, 204-215 (2019).

28. Szegedi V, et al. Robust perisomatic GABAergic self-innervation inhibits basket cells in the human and mouse supragranular neocortex. Elife 9, (2020). 
29. Menendez de la Prida L, Benavides-Piccione R, Sola R, Pozo MA. Electrophysiological properties of interneurons from intraoperative spiking areas of epileptic human temporal neocortex. Neuroreport 13, 1421-1425 (2002).

30. Jiang $\mathrm{M}$, et al. Enhancement of asynchronous release from fast-spiking interneuron in human and rat epileptic neocortex. PLoS Biol 10, e1001324 (2012).

31. Wang B, et al. Firing Frequency Maxima of Fast-Spiking Neurons in Human, Monkey, and Mouse Neocortex. Front Cell Neurosci 10, 239 (2016).

32. Chen W, Zhang JJ, Hu GY, Wu CP. Electrophysiological and morphological properties of pyramidal and nonpyramidal neurons in the cat motor cortex in vitro. Neuroscience 73 , 39-55 (1996).

33. Povysheva NV, Zaitsev AV, Rotaru DC, Gonzalez-Burgos G, Lewis DA, Krimer LS. Parvalbumin-positive basket interneurons in monkey and rat prefrontal cortex. J Neurophysiol 100, 2348-2360 (2008).

34. Rudy B, Fishell G, Lee S, Hjerling-Leffler J. Three groups of interneurons account for nearly $100 \%$ of neocortical GABAergic neurons. Dev Neurobiol 71, 45-61 (2011).

35. Lim L, Mi D, Llorca A, Marin O. Development and Functional Diversification of Cortical Interneurons. Neuron 100, 294-313 (2018).

36. Goldberg EM, Jeong HY, Kruglikov I, Tremblay R, Lazarenko RM, Rudy B. Rapid developmental maturation of neocortical FS cell intrinsic excitability. Cereb Cortex 21, 666682 (2011).

37. Povysheva NV, Zaitsev AV, Gonzalez-Burgos G, Lewis DA. Electrophysiological heterogeneity of fast-spiking interneurons: chandelier versus basket cells. PLoS One 8, e70553 (2013).

38. Albertson AJ, Bohannon AS, Hablitz JJ. HCN Channel Modulation of Synaptic Integration in GABAergic Interneurons in Malformed Rat Neocortex. Front Cell Neurosci 11, 109 (2017).

39. Willems JGP, Wadman WJ, Cappaert NLM. Parvalbumin interneuron mediated feedforward inhibition controls signal output in the deep layers of the perirhinal-entorhinal cortex. Hippocampus 28, 281-296 (2018).

40. Aponte $\mathrm{Y}$, Lien CC, Reisinger $\mathrm{E}$, Jonas $\mathrm{P}$. Hyperpolarization-activated cation channels in fast-spiking interneurons of rat hippocampus. J Physiol 574, 229-243 (2006).

41. Shah MM. Cortical HCN channels: function, trafficking and plasticity. J Physiol 592, 2711-2719 (2014). 
42. Santoro B, Shah MM. Hyperpolarization-Activated Cyclic Nucleotide-Gated Channels as Drug Targets for Neurological Disorders. Annu Rev Pharmacol Toxicol 60, 109-131 (2020).

43. Yang SS, Li YC, Coley AA, Chamberlin LA, Yu P, Gao WJ. Cell-Type Specific Development of the Hyperpolarization-Activated Current, Ih, in Prefrontal Cortical Neurons. Front Synaptic Neurosci 10, 7 (2018).

44. Pelkey KA, Chittajallu R, Craig MT, Tricoire L, Wester JC, McBain CJ. Hippocampal GABAergic Inhibitory Interneurons. Physiol Rev 97, 1619-1747 (2017).

45. Notomi T, Shigemoto R. Immunohistochemical localization of Ih channel subunits, HCN1-4, in the rat brain. J Comp Neurol 471, 241-276 (2004).

46. Southan AP, Morris NP, Stephens GJ, Robertson B. Hyperpolarization-activated currents in presynaptic terminals of mouse cerebellar basket cells. J Physiol 526 Pt 1, 91-97 (2000).

47. Varga $\mathrm{V}$, et al. The presence of pacemaker $\mathrm{HCN}$ channels identifies theta rhythmic GABAergic neurons in the medial septum. J Physiol 586, 3893-3915 (2008).

48. Nusser Z. Variability in the subcellular distribution of ion channels increases neuronal diversity. Trends Neurosci 32, 267-274 (2009).

49. Elgueta C, Kohler J, Bartos M. Persistent discharges in dentate gyrus perisomainhibiting interneurons require hyperpolarization-activated cyclic nucleotide-gated channel activation. J Neurosci 35, 4131-4139 (2015).

50. Jimenez-Sosa MS. Fast spiking GABAergic interneurons in the dorsolateral prefrontal cortex of non-human primate: Comparison study to mice and human, with focus on subthreshold intrinsic properties. Electronic Thesis and Dissertation Repository 7228, (2020).

51. Petersen CC, Crochet S. Synaptic computation and sensory processing in neocortical layer 2/3. Neuron 78, 28-48 (2013).

52. Debanne D, Inglebert $Y$, Russier M. Plasticity of intrinsic neuronal excitability. Curr Opin Neurobiol 54, 73-82 (2019).

53. Williams SR, Stuart GJ. Site independence of EPSP time course is mediated by dendritic I(h) in neocortical pyramidal neurons. J Neurophysiol 83, 3177-3182 (2000).

54. Jouhanneau JS, Kremkow J, Poulet JFA. Single synaptic inputs drive high-precision action potentials in parvalbumin expressing GABA-ergic cortical neurons in vivo. Nat Commun 9, 1540 (2018).

55. Ishii TM, Takano M, Ohmori H. Determinants of activation kinetics in mammalian hyperpolarization-activated cation channels. J Physiol 537, 93-100 (2001). 
56. Foldy C, Lee SH, Morgan RJ, Soltesz I. Regulation of fast-spiking basket cell synapses by the chloride channel CIC-2. Nat Neurosci 13, 1047-1049 (2010).

57. Krienen FM, et al. Innovations present in the primate interneuron repertoire. Nature $586,262-269$ (2020).

58. Kumar S, Stecher G, Suleski M, Hedges SB. TimeTree: A Resource for Timelines, Timetrees, and Divergence Times. Mol Biol Evol 34, 1812-1819 (2017).

59. D'Angelo $E$, et al. Realistic modeling of neurons and networks: towards brain simulation. Funct Neurol 28, 153-166 (2013).

60. Eyal G, et al. Human Cortical Pyramidal Neurons: From Spines to Spikes via Models. Front Cell Neurosci 12, 181 (2018).

61. Pals $M$, Stewart TC, Akyurek EG, Borst JP. A functional spiking-neuron model of activity-silent working memory in humans based on calcium-mediated short-term synaptic plasticity. PLoS Comput Biol 16, e1007936 (2020).

62. Heng Kang Yao AG-M, Frank Mazza, Homeira Moradi Chameh, Thomas D. Prevot, John Griffiths, Shreejoy J. Tripathy, Taufik A. Valiante, Etienne Sibille, Etay Hay. Reduced inhibition in depression impairs stimulus processing in human cortical microcircuits. bioRxiv, (2021). 
Figure 1
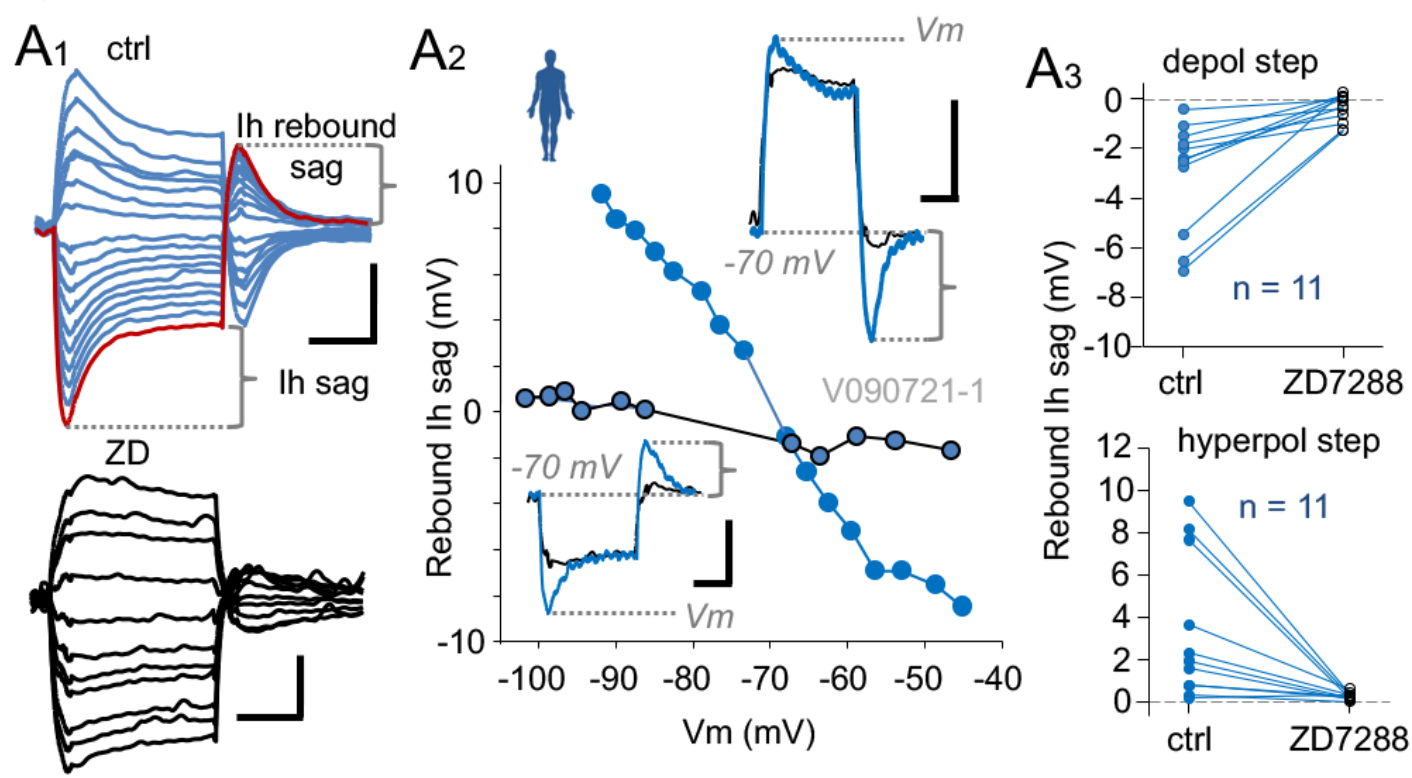

B1 In sag from -70 to $-90 \mathrm{mV}$

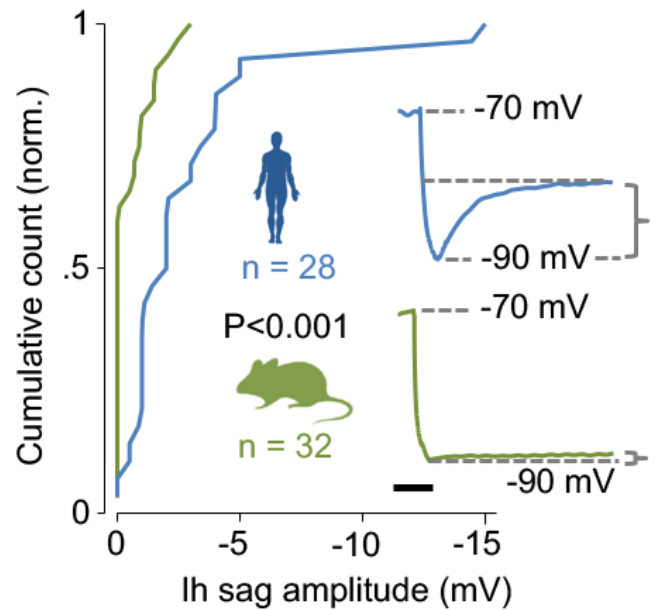

$\mathrm{C}_{1}$

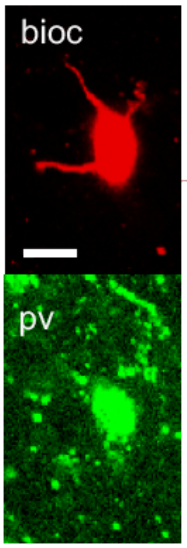

B2 Ih sag from -50 to $-70 \mathrm{mV}$

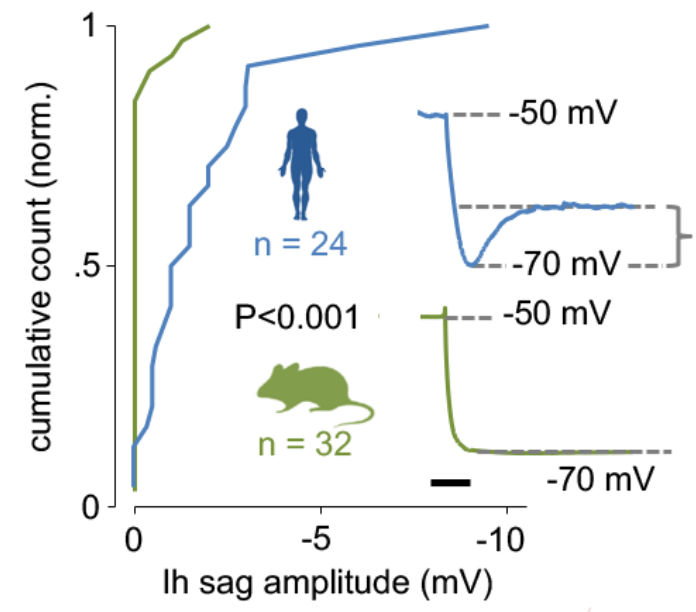

$\mathrm{C}_{2}$

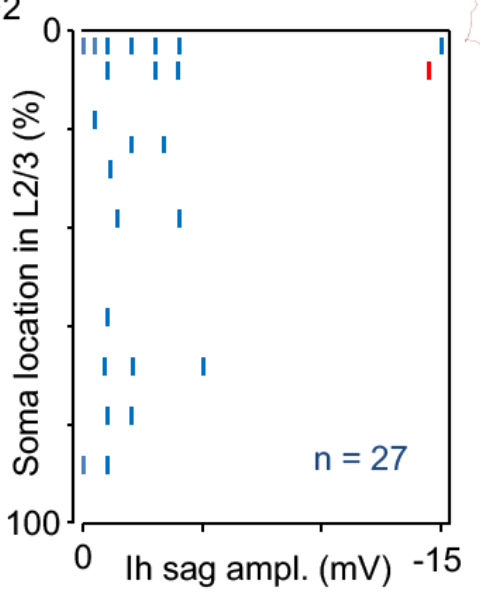

L1
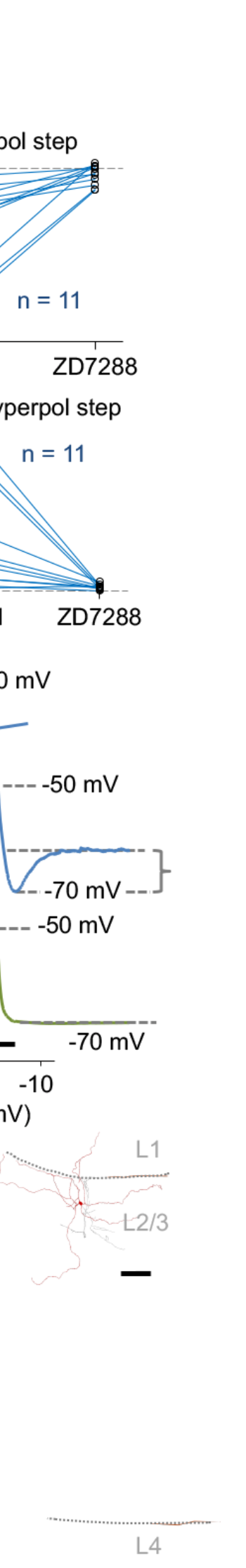
Figure 2
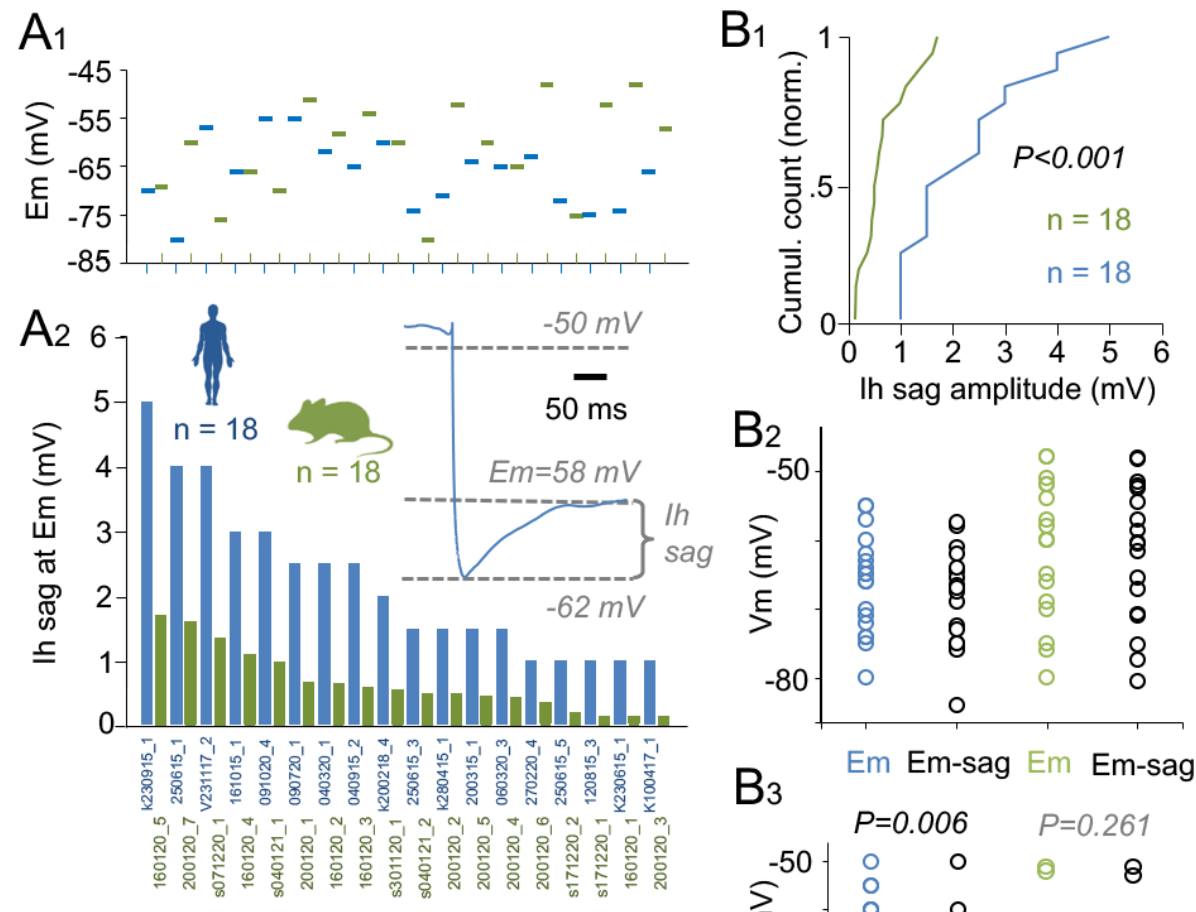

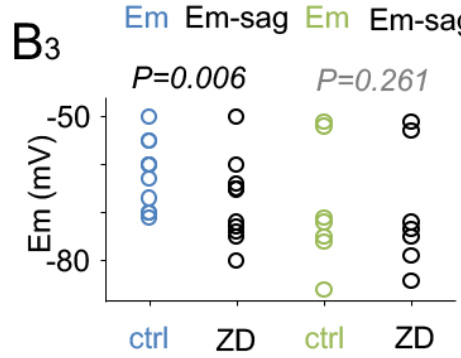


Figure 3
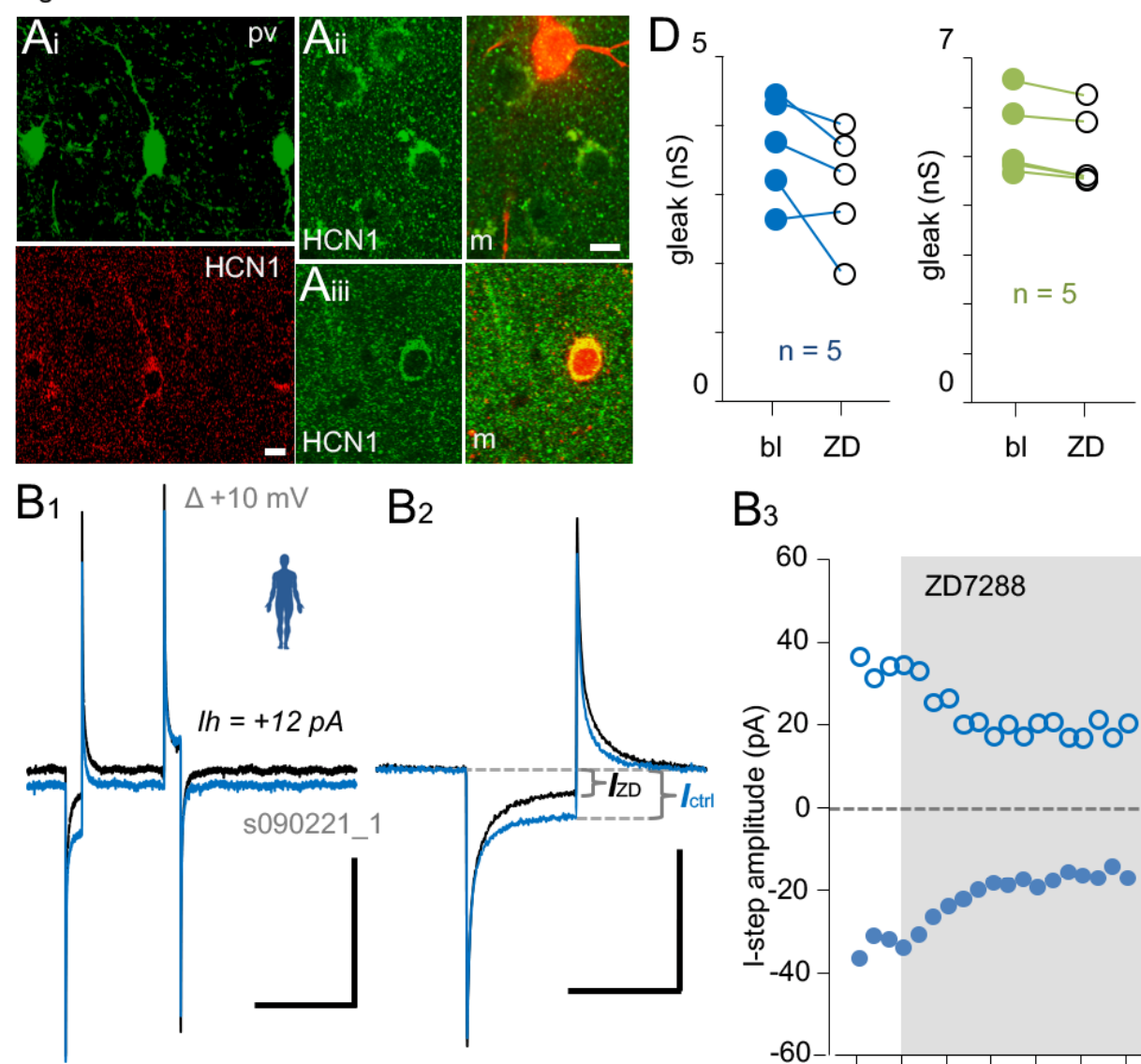

$\mathrm{B}_{3}$
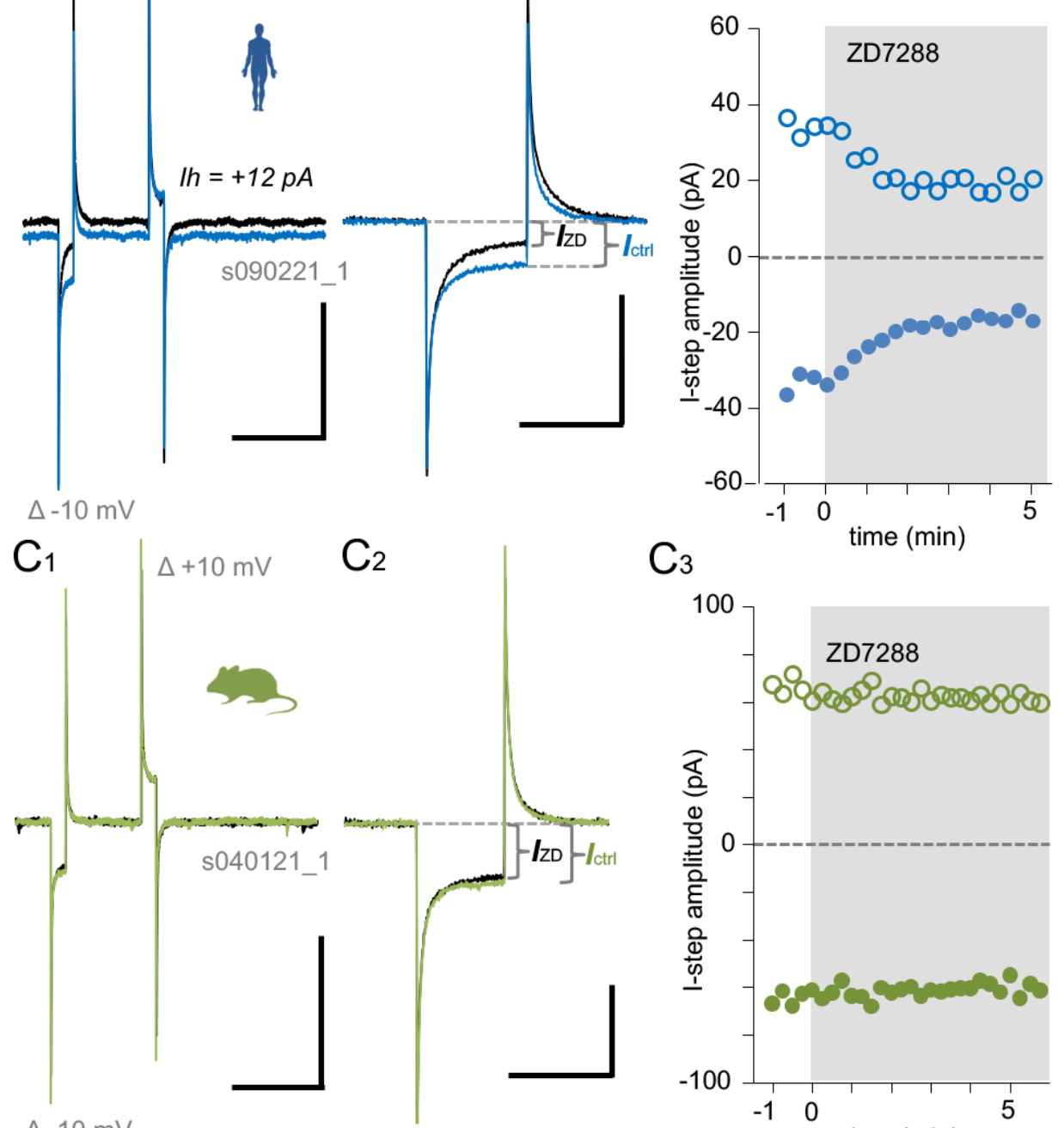

$\mathrm{C}_{3}$

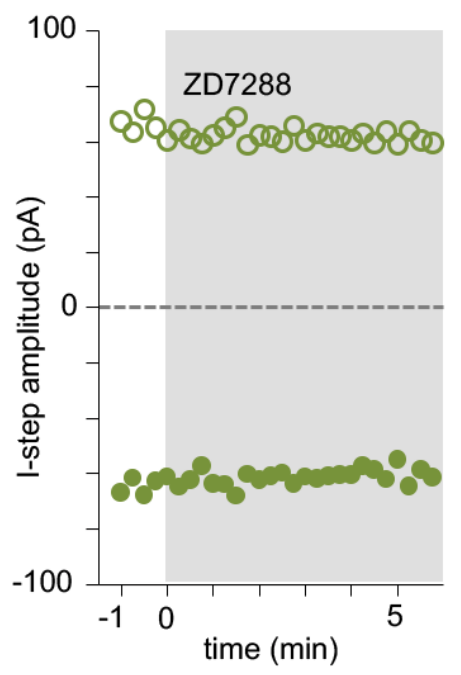


Figure 4

$\mathrm{A}_{1}$ Istep

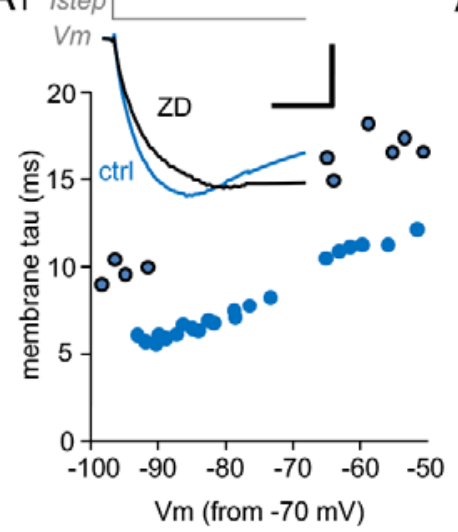

$\mathrm{B}_{1}$

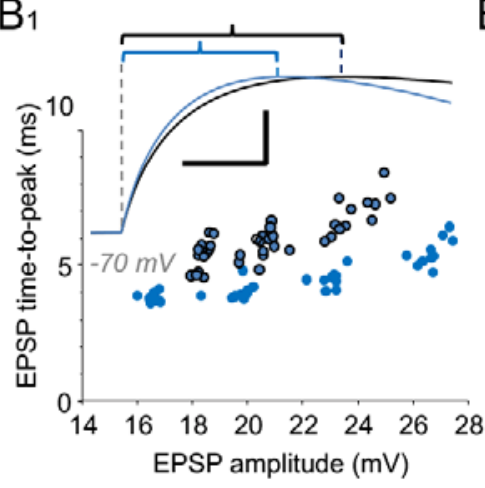

$\mathrm{A}_{2}$

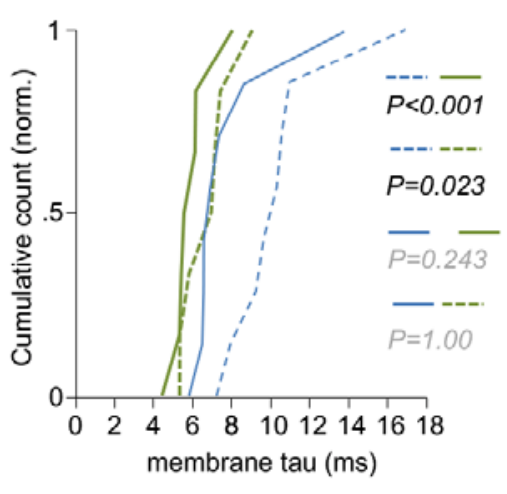

$\mathrm{B}_{2}$

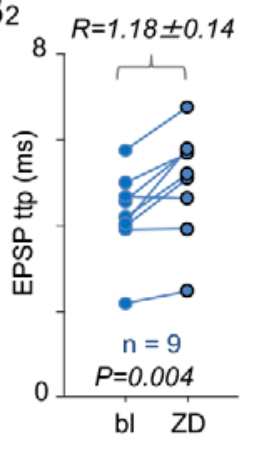

$\mathrm{A}_{3}$

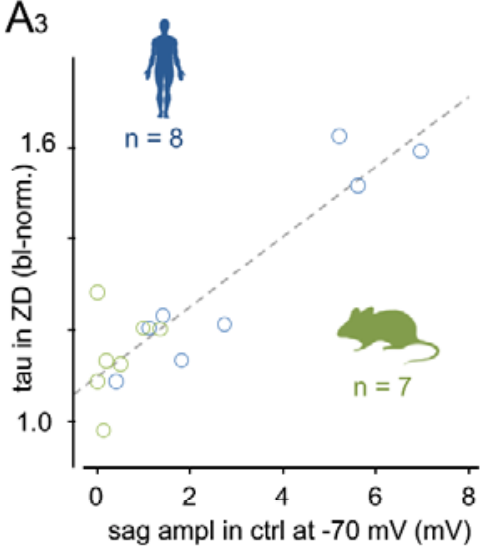

$\mathrm{B} 3$

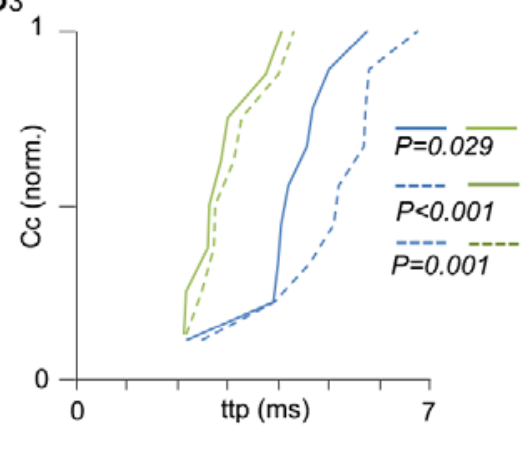


Figure 5.
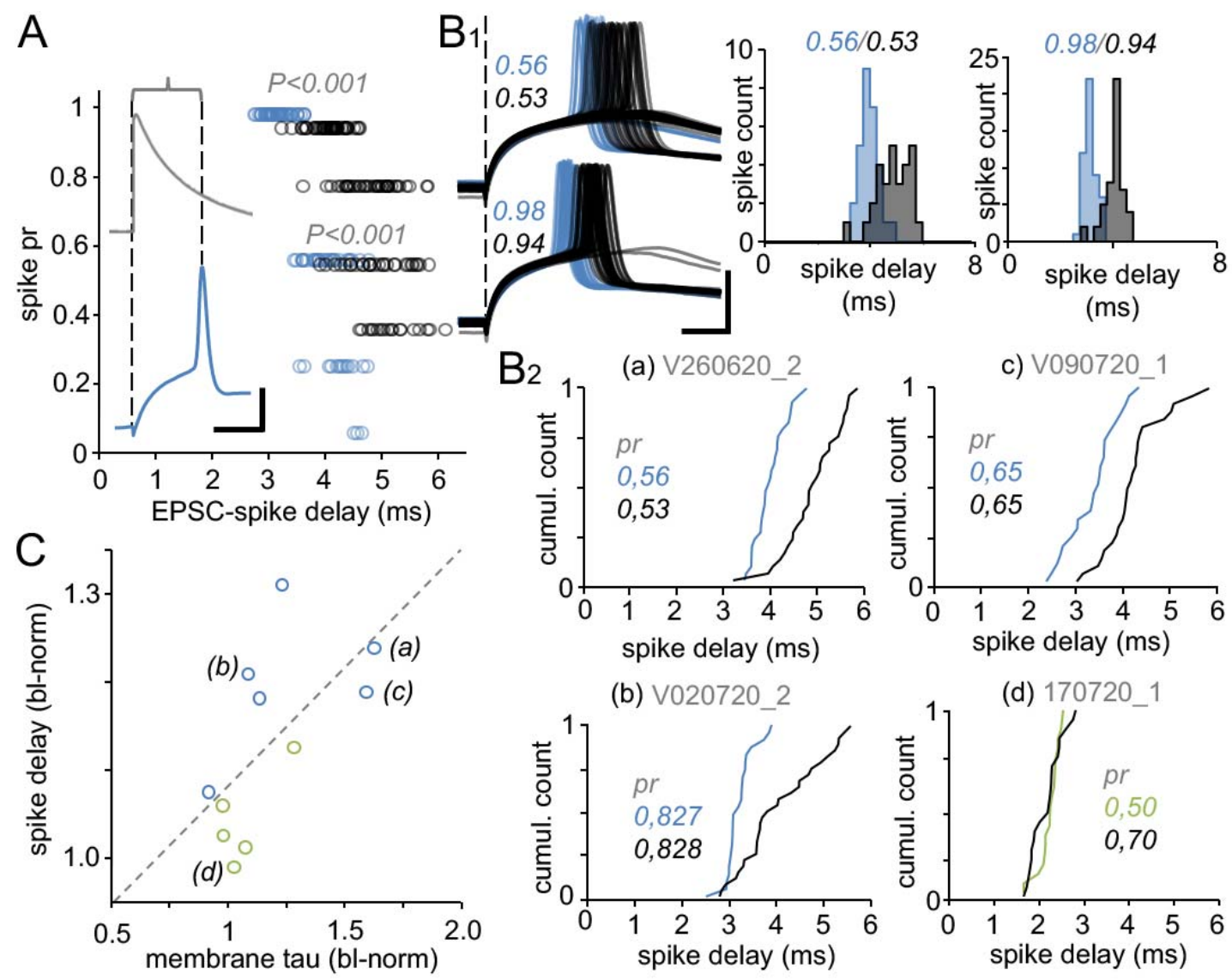

(ms) $\quad(\mathrm{ms})$

(b) V020720 2

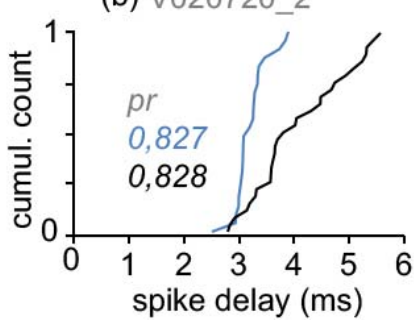

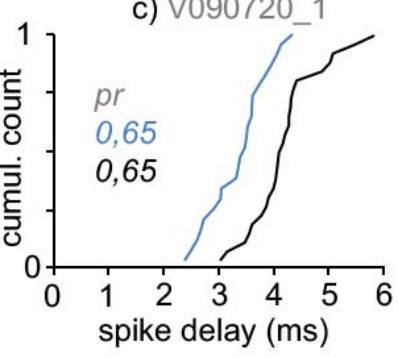

(d) 170720_1

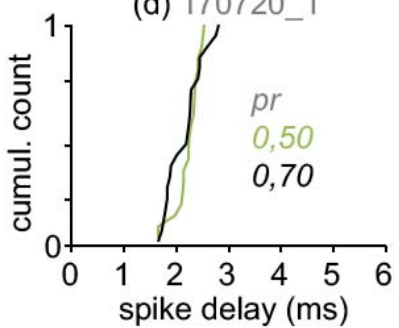


bioRxiv preprint doi: https://doi.org/10.1101/2021.06.09.447671; this version posted June 10, 2021. The copyright holder for this preprint (which was not certified by peer review) is the author/funder, who has granted bioRxiv a license to display the preprint in perpetuity. It is made available under aCC-BY 4.0 International license.

Figure 6.
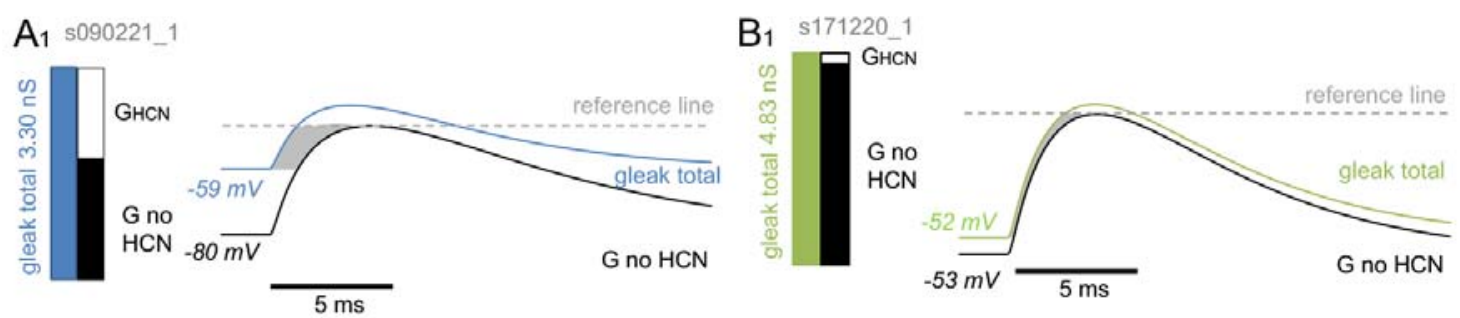

A2 s101220_1

B2 s040121_1
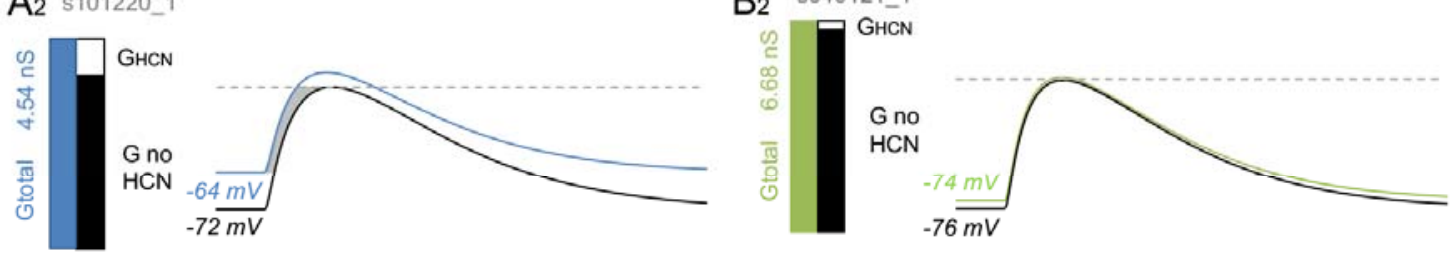

A 3 V100221_1
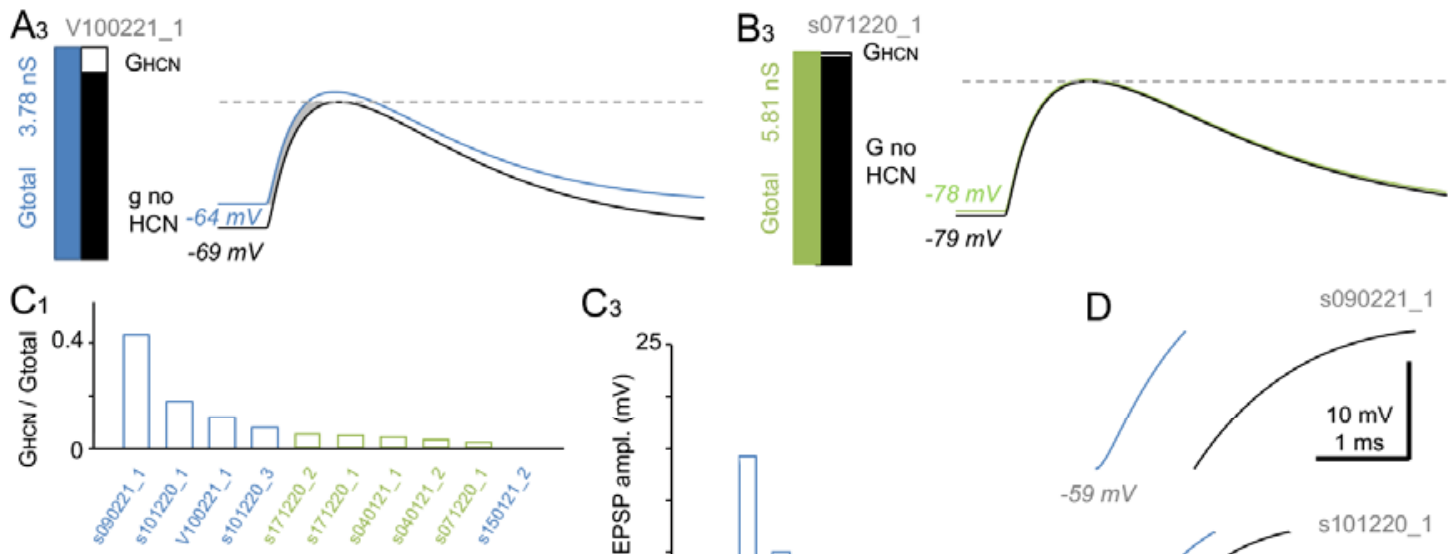

$\mathrm{C}_{3}$
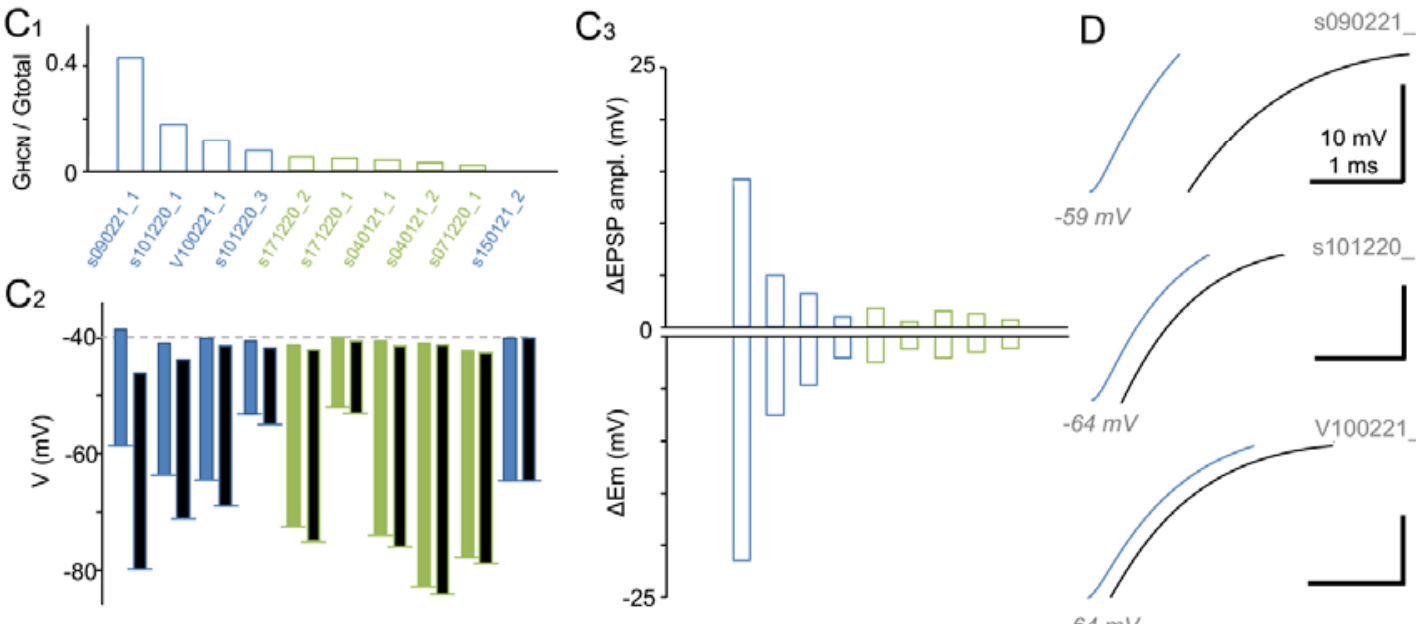

$-59 m V$
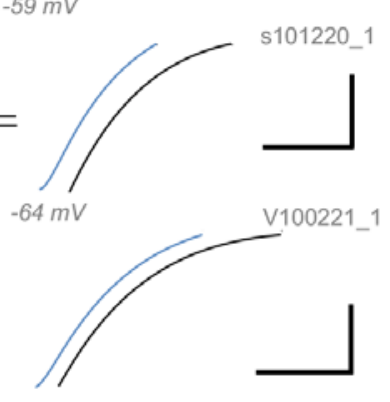
Supplementary figure $\mathrm{S} 1$
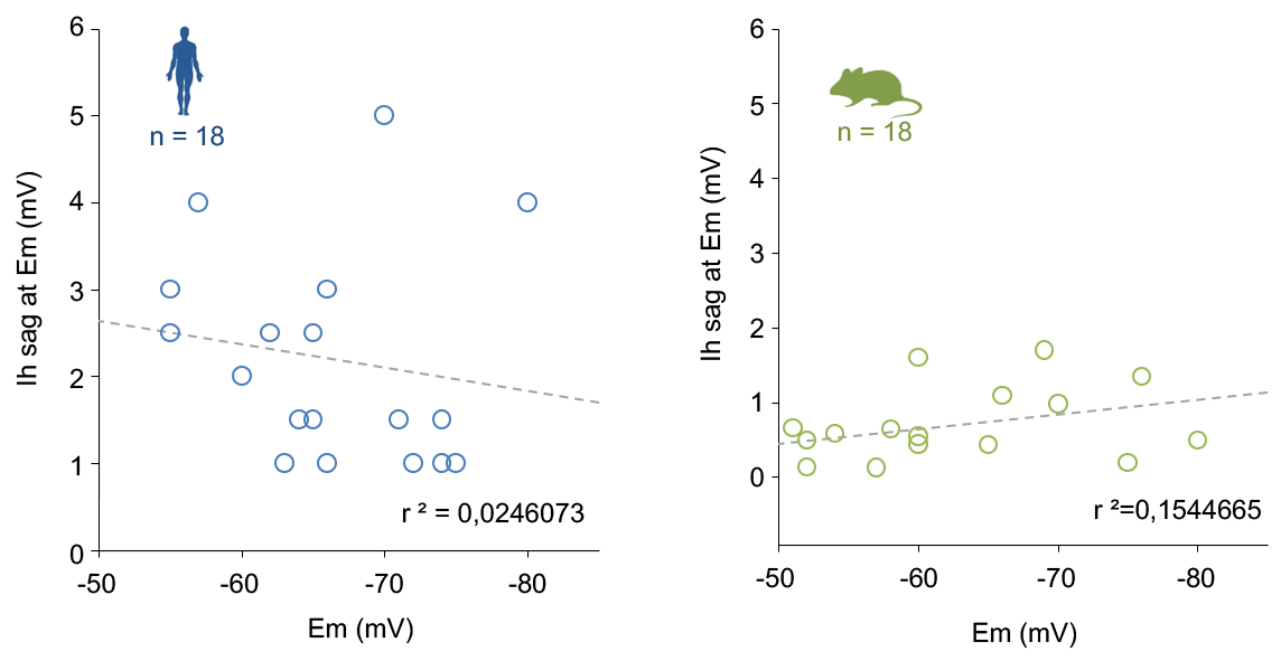

Supplementary figure S2
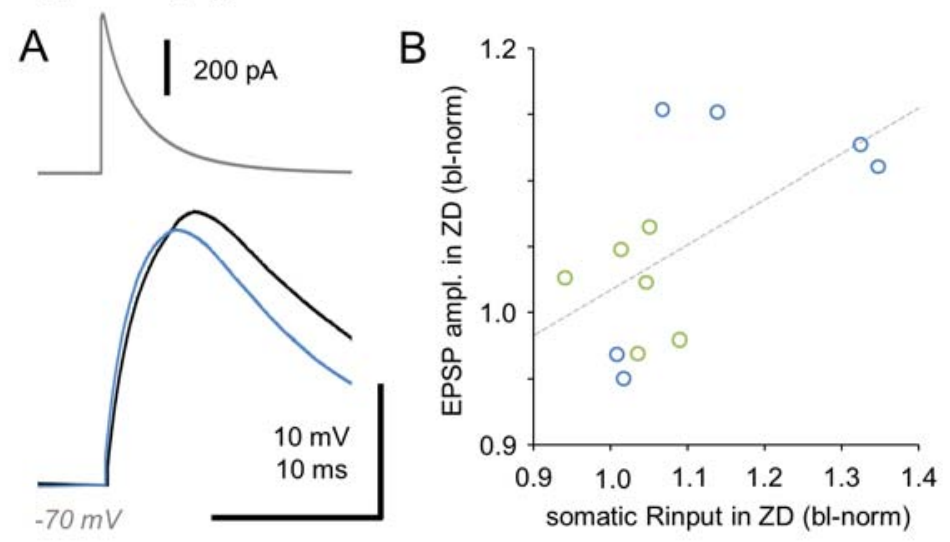

Supplemental figure S3

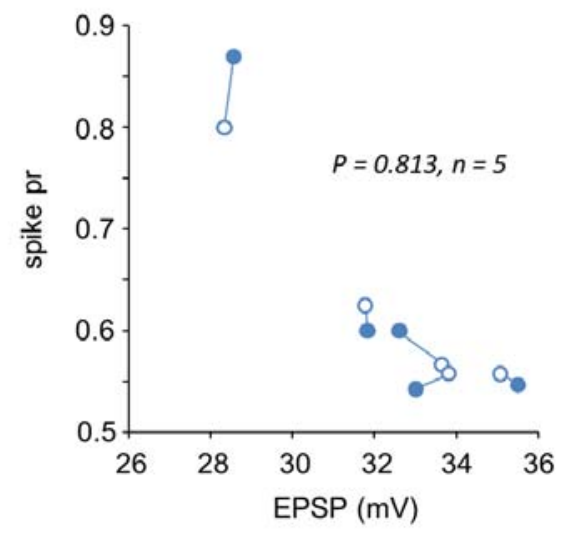

